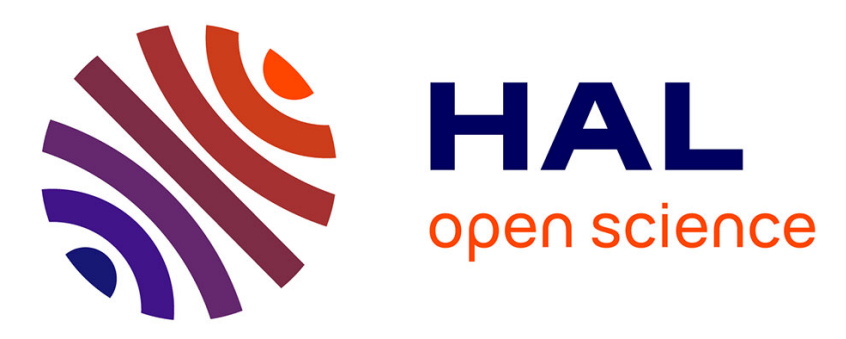

\title{
Algebraic disturbances and their consequences in rotating channel flow transition
}

Sharath Jose, Vishnu Kuzhimparampil, Benoît Pier, Rama Govindarajan

\section{To cite this version:}

Sharath Jose, Vishnu Kuzhimparampil, Benoît Pier, Rama Govindarajan. Algebraic disturbances and their consequences in rotating channel flow transition. Physical Review Fluids, 2017, 2 (8), pp.083901. 10.1103/PhysRevFluids.2.083901 . hal-01578604

\section{HAL Id: hal-01578604 https://hal.science/hal-01578604}

Submitted on 29 Aug 2017

HAL is a multi-disciplinary open access archive for the deposit and dissemination of scientific research documents, whether they are published or not. The documents may come from teaching and research institutions in France or abroad, or from public or private research centers.
L'archive ouverte pluridisciplinaire HAL, est destinée au dépôt et à la diffusion de documents scientifiques de niveau recherche, publiés ou non, émanant des établissements d'enseignement et de recherche français ou étrangers, des laboratoires publics ou privés. 


\title{
Algebraic disturbances and their consequences in rotating channel flow transition
}

\author{
Sharath Jose, ${ }^{1, *}$ Vishnu Kuzhimparampil, ${ }^{1}$ Benoît Pier, ${ }^{2}$ and Rama Govindarajan ${ }^{3}$ \\ ${ }^{1}$ TIFR Centre for Interdisciplinary Sciences, Tata Institute of Fundamental Research, \\ 21 Brundavan Colony, Narsingi, Hyderabad 500075, India \\ ${ }^{2}$ Laboratoire de mécanique des fluides et d'acoustique, CNRS-École centrale de Lyon-Université \\ Claude-Bernard Lyon 1-INSA, 36 avenue Guy-de-Collongue, F-69134 Écully, France \\ ${ }^{3}$ International Centre for Theoretical Sciences, Tata Institute of Fundamental Research, Survey 151, \\ Shivakote, Hesaraghatta Hobli, Bengaluru 560089, India \\ (Received 19 September 2016; published 14 August 2017)
}

\begin{abstract}
It is now established that subcritical mechanisms play a crucial role in the transition to turbulence of nonrotating plane shear flows. The role of these mechanisms in rotating channel flow is examined here in the linear and nonlinear stages. Distinct patterns of behavior are found: the transient growth leading to nonlinearity at low rotation rates $R o$, a highly chaotic intermediate $R o$ regime, a localized weak chaos at higher $R o$, and complete stabilization of transient disturbances at very high $R o$. At very low $R o$, the transient growth amplitudes are close to those for nonrotating flow, but Coriolis forces assert themselves by producing distinct asymmetry about the channel centreline. Nonlinear processes are then triggered, in a streak-breakdown mode of transition. The high Ro regimes do not show these signatures; here the leading eigenmode emerges as dominant in the early stages. Elongated structures plastered close to one wall are seen at higher rotation rates. Rotation is shown to reduce nonnormality in the linear operator, in an indirect manifestation of Taylor-Proudman effects. Although the critical Reynolds for exponential growth of instabilities is known to vary a lot with rotation rate, we show that the energy critical Reynolds number is insensitive to rotation rate. It is hoped that these findings will motivate experimental verification and examination of other rotating flows in this light.
\end{abstract}

DOI: 10.1103/PhysRevFluids.2.083901

\section{INTRODUCTION}

Rotation of the system, in a number of flow situations, plays an important role in stability and turbulence characteristics. Rotational effects are seen to influence the evolution of several flow phenomena of practical interest ranging from engineering to geophysics. Atmospheric and oceanic flows offer a myriad of not entirely understood phenomena, which are affected by Earth's rotation in addition to other physics [1,2]. In industrial situations, the modeling of rotational effects of flows is a crucial aspect in the design procedure of several technologies: pumps and turbines, for example [3].

The effect of rotation on shear flow instabilities is not immediately obvious, and it depends largely on the strength of the rotation. Rotation introduces a body force which is a function of space and time, and bears some analogy to density stratification. At high Ro, the flow is expected to obey Taylor-Proudman behavior [4,5], by which variations parallel to the rotation axis are strongly suppressed. In the manner of swirling flows, an inviscid criterion for instability in parallel flows of the form $\boldsymbol{U}=[U(y), 0,0]$, with the rotation vector $\boldsymbol{\Omega}=(0,0, \Omega)$ perpendicular to the plane of the flow, was formulated by Bradshaw [6] and Pedley [7]. As per the criterion, an instability can occur if at any point in the flow the absolute vorticity of the base flow and the rotation vector are antiparallel. Subsequent studies have shown that this simple analogue of the

\footnotetext{
*josesk@ tifrh.res.in
} 
Rayleigh criterion provides good predictions in many parallel flows even in the presence of viscous effects [8-12].

One of the most commonly studied systems is the pressure-driven flow between two stationary, parallel plates that is rotated about the spanwise coordinate, which is also the geometry of our interest. This is an appealing system for investigation as it is a simple rotating shear flow which offers regions that are both stable and unstable as per the inviscid criterion given above. Henceforth, this system will be referred to simply as rotating channel flow. This flow is characterized by two parameters, the Reynolds number $R e=U_{0} d / v$ and the rotation number $R o=\Omega d / U_{0}$, where $U_{0}$ is the centerline velocity in the channel, $d$ is its half-width, $\Omega$ is the rotation rate, and $v$ is the kinematic viscosity of the fluid.

It was found experimentally that the critical Reynolds number $R e_{c r}$, below which no exponential instabilities exist, may be up to two orders magnitude lower than that of a nonrotating channel $[8,11]$. This critical Reynolds number shows a nonmonotonic variation with the strength of rotation and is very sensitive to it. Just past $R e_{c r}$, the first unstable mode corresponds to a stationary streamwise-invariant disturbance. As we move further into the unstable part of the parameter space, we may find oblique modes that have growth rates comparable to the streamwise-invariant mode [13]. At high $R o$, Taylor-Proudman behavior sets in and these streamwise-invariant rotation modes are suppressed. The two-dimensional spanwise-invariant Tollmien-Schlichting (TS) mode can be triggered even for values of $R e$ above the critical value 5772 for nonrotating channels $[14,15]$. But in the regime where both the TS mode and the rotation mode are present, the rotation mode is expected to win over due to a much larger growth rate [13].

Secondary instabilities of the traveling wave type with short and long wavelengths which eventually broke down to turbulence had been observed in experiments [11]. Merging and splitting of vortex pairs through a nonlinear wavelength selection process was also seen. These types of motions were further confirmed by numerical studies [16-18]. Matsubara and Alfredsson attribute the secondary instability to the spanwise inflectional profile resulting from the saturation of the primary disturbance [19]. The existence of secondary and tertiary saturated solutions of rotating shear flows has also been investigated [13,20,21]. The turbulent rotating channel flow has been studied extensively for a wide range of Reynolds numbers through experiments and simulations $[9,22-25]$.

For the rotating channel flow, the effect of nonmodal (algebraic) growth of disturbances has not been previously investigated to our knowledge, despite extensive studies on instabilities and turbulence in the system. In a different geometry, namely on the rotating asymptotic suction boundary layer, transient growth effects were studied [26]. The effect of subcritical mechanisms in nonmagnetized accretion disks modeled as plane shear flows with rotation have been studied [27-29]. Given that it has been firmly established that subcritical linear processes arising from nonmodal dynamics play a pivotal role in determining the conditions of transition to turbulence in a variety of shear flows $[30,31]$, it is important to ask how rotation will modify the role of nonmodal dynamics.

In some range of rotation numbers, the critical Reynolds number is very low and exponential growth of instabilities may be expected to dominate. However, given that the rotation number is independent of the Reynolds number and that the critical Reynolds number is a sensitive function of $R o$, it is necessary to investigate algebraic growth in the modally stable region of the $R e$-Ro plane. Second, in the unstable region as well, algebraic growth can affect the transition process in spite of coexisting exponential modes of growth. In this article, we seek to characterize the role played by algebraically growing disturbances in different rotation regimes, modally stable and unstable, within the purview of the transition processes.

\section{SYSTEM}

Our system, consisting of a pressure driven flow between parallel fixed walls, is subjected to rotation about the spanwise direction with a constant angular velocity $\boldsymbol{\Omega}=(0,0, \Omega)$ (Fig. 1). For the 


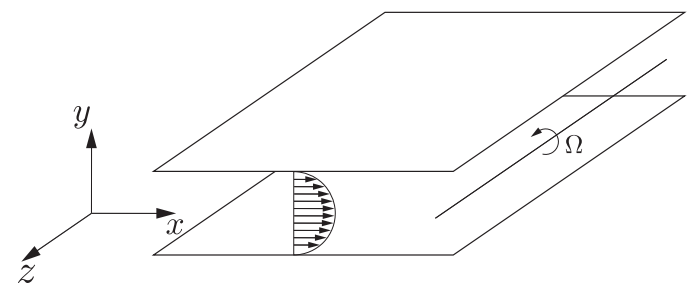

FIG. 1. The rotating channel, with a parabolic streamwise velocity $\left(U=1-y^{2}\right)$ and a rotation rate $\Omega$ about the spanwise coordinate.

purposes of the analysis to follow, we consider the two parallel walls to extend infinitely; i.e., there are no end walls. It is also convenient to work in a frame of reference that is rotating along with the channel. Then the governing equations for the velocity $\boldsymbol{u}^{*}=\left(u^{*}, v^{*}, w^{*}\right)$ and pressure $p^{*}$ are the incompressible Navier-Stokes equations in the rotating frame given by

$$
\begin{gathered}
\partial_{t^{*}} \boldsymbol{u}^{*}+\boldsymbol{u}^{*} \cdot \nabla^{*} \boldsymbol{u}^{*}=-\frac{1}{\rho} \nabla^{*} p^{*}+v \Delta^{*} \boldsymbol{u}^{*}-2 \boldsymbol{\Omega} \times \boldsymbol{u}^{*}, \\
\nabla^{*} \cdot \boldsymbol{u}^{*}=0 .
\end{gathered}
$$

Here $\rho$ is constant density of the fluid. The centrifugal force has been absorbed into the pressure term. With $U_{0}$ and $d$ as the velocity and length scales respectively, the Reynolds and rotation numbers as defined in the previous section, and $\hat{z}$ being the unit vector along the spanwise coordinate, the governing equations in the nondimensional form are as follows:

$$
\begin{gathered}
\partial_{t} \boldsymbol{u}+\boldsymbol{u} \cdot \nabla \boldsymbol{u}=-\nabla p+\frac{1}{R e} \Delta \boldsymbol{u}-2 R o \hat{z} \times \boldsymbol{u}, \\
\nabla \cdot \boldsymbol{u}=0 .
\end{gathered}
$$

When the effects of the end walls are neglected, the base flow adopts a parabolic streamwise velocity profile $U=\left(1-y^{2}\right)$ [10]. Note that we use uppercase to denote base flow. The transverse and wall-normal velocity components are zero. In a real system with end walls, a secondary flow in the form of a double vortex is set up [32]. As is standard for rotating flows, a mean pressure gradient is sustained in the wall-normal direction $y$, balancing Coriolis forces, and may be obtained from Eq. (3) as

$$
\partial_{y} P=-2 U R o \text {. }
$$

Tritton and Davies [33], using an elegant displaced particle argument, describe the instability mechanism in rotating plane shear flows in terms of the imbalance of centrifugal force and force due to this wall-normal pressure gradient. Their argument recovers the inviscid criterion derived by Bradshaw [6] and Pedley [7]. In nondimensionalized form, the criterion for instability has the following form:

$$
\phi(y)=2 R o\left(-\frac{\partial U}{\partial y}+2 R o\right)<0 .
$$

Examining our system in this context, we see that for a given sense of rotation, one side of the channel is inviscidly stable and the other is unstable. Since we have a base flow which is symmetric about the centerline, if the sense of rotation were to be reversed, we would merely have a switch in which side is unstable, and all results would merely be mirror images. We therefore fix our rotation to be anticyclonic, i.e., $R o>0$. However, we caution that for asymmetric shear flows, positive and negative $R o$ would need to be studied separately [26,33,34]. 


\section{LINEAR ANALYSIS}

\section{A. Governing equations and methodology}

On introducing perturbations that are nominally small compared to the base state quantities, and by linearizing the governing equations, we can study linear stability characteristics of the base flow. In the current setting where the channel extends infinitely in the streamwise and the spanwise directions, we can consider the disturbances to be periodic in these directions with a specific wavenumber $\boldsymbol{k}=(\alpha, \beta)$, with $\alpha$ the streamwise wavenumber and $\beta$ the spanwise wavenumber, so disturbances take on the form $f=\hat{f}(y, t) e^{i(\alpha x+\beta z)}$. In terms of the wall-normal velocity disturbance $\left[v=\hat{v}(y, t) e^{i(\alpha x+\beta z)}\right]$ and the wall-normal vorticity disturbance $\left[\eta=\hat{\eta}(y, t) e^{i(\alpha x+\beta z)}\right]$, the resulting system of linear equations is

$$
\begin{gathered}
\frac{\partial \hat{\boldsymbol{q}}}{\partial t}=\mathbf{L} \hat{\boldsymbol{q}}, \quad \hat{\boldsymbol{q}}(t=0)=\hat{\boldsymbol{q}}_{0}, \\
\text { where } \hat{\boldsymbol{q}}=\left[\begin{array}{l}
\hat{v} \\
\hat{\eta}
\end{array}\right], \quad \text { and } \mathbf{L}=\left[\begin{array}{cc}
D^{2}-k^{2} & 0 \\
0 & 1
\end{array}\right]^{-1}\left[\begin{array}{cc}
L_{o s} & -2 i R o \beta \\
-i \beta U^{\prime}+2 i R o \beta & L_{s q}
\end{array}\right] .
\end{gathered}
$$

Here $D()=.\partial(.) / \partial y$, a prime denotes $d(.) / d y$, and $k^{2}=\alpha^{2}+\beta^{2} . L_{O S}$ and $L_{S Q}$ are the OrrSommerfeld and Squire operators given by

$$
\begin{gathered}
L_{O S}=i \alpha U^{\prime \prime}-i \alpha U\left(D^{2}-k^{2}\right)+\frac{1}{R e}\left(D^{2}-k^{2}\right)^{2}, \\
L_{S Q}=-i \alpha U+\frac{1}{\operatorname{Re}}\left(D^{2}-k^{2}\right) .
\end{gathered}
$$

The perturbation pressure can then be obtained as the solution of a Poisson equation. The boundary conditions for the above system of equations are

$$
\hat{v}( \pm 1, t)=D \hat{v}( \pm 1, t)=\hat{\eta}( \pm 1, t)=0 .
$$

For the rest of the article, we simply refer to wall-normal components of velocity and vorticity as normal components unless suggested otherwise.

If the spectrum of the linearized operator $\mathbf{L}$ in Eq. (7) contains an eigenvalue with a positive real part, then there is an exponentially growing mode that causes the base flow to transition to another state. If there is no such growing eigenmode, we may conclude that the flow is asymptotically stable. In the present system, exponential instabilities are known to set in fairly low values of $R e$.

The linearized problem governed by Eq. (7) may also be addressed as an initial value problem with a view of finding the initial condition that maximizes an objective functional, i.e., in this case the disturbance kinetic energy [30]. The maximum possible gain at a given time $G(t)$ and its global maximum $G_{\max }$ can be defined for a set of fixed values of parameters $R e$ and $R o$ as follows:

$$
G(t ; \alpha, \beta)=\sup _{\hat{\boldsymbol{q}}_{0}} \frac{\|\hat{\boldsymbol{q}}(t)\|_{E}^{2}}{\left\|\hat{\boldsymbol{q}}_{0}\right\|_{E}^{2}} \quad \text { and } G_{\max }=\sup _{t \geqslant 0, \alpha, \beta} G(t ; \alpha, \beta) .
$$

The disturbance kinetic energy norm that is to be maximized is defined in terms of the normal velocity $v$ and normal vorticity $\eta$ as

$$
\|\hat{\boldsymbol{q}}(t)\|_{E}^{2}=\frac{1}{2 k^{2}} \int_{-1}^{1} \hat{\boldsymbol{q}}^{H}(t)\left[\begin{array}{cc}
k^{2}-D^{2} & 0 \\
0 & 1
\end{array}\right] \hat{\boldsymbol{q}}(t) d y .
$$

For a given $R e$ and $R o$, the streamwise wavenumber $\alpha$, spanwise wavenumber $\beta$, and time $t$ that yield $G_{\max }$ are said to be optimal for maximizing the disturbance energy. These values are denoted as the optimal streamwise wavenumber $\alpha_{\text {opt }}$, optimal spanwise wavenumber $\beta_{\text {opt }}$, and optimal time $T_{\mathrm{opt}} \cdot$ 


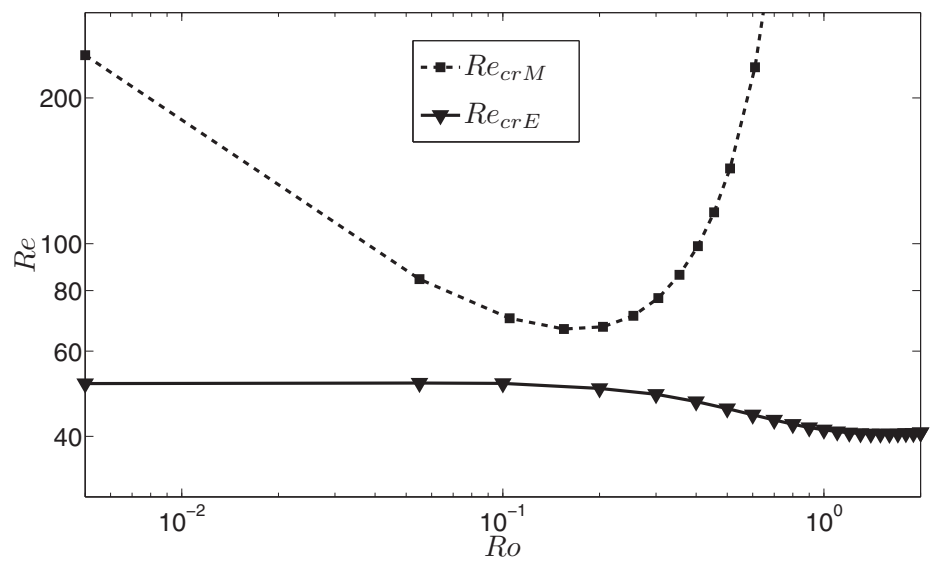

FIG. 2. The stability boundaries as per the energy and the eigenvalue methods.

For obtaining the optimal initial condition, the eigenvectors of the linearized operators can be used as a basis as they are complete in the present bounded geometry [35]. Upon inspecting the pseudospectra of the linearized operator, we find that a resolution of $N=65$ Chebyshev collocation points in the normal direction is sufficient to form a complete basis of eigenvectors. Calculations performed with $N=81$ or 121 produce the same result up to at least nine decimal places. Defining $\boldsymbol{\Lambda}$ as the diagonal matrix consisting of eigenvalues of the operator $\mathbf{L}$, and $\tilde{\mathbf{q}}$ as the corresponding set of eigenvectors of $\mathbf{L}$, we may express solutions of Eq. (7) in variable separable form as

$$
\hat{\boldsymbol{q}}(y, t)=\tilde{\mathbf{q}}(y) \boldsymbol{k}(t) .
$$

This allows us to deal with a computationally simpler problem as we now have $\boldsymbol{k}(t)=e^{\boldsymbol{\Lambda} t} \boldsymbol{k}(0)$. Eigenfunctions which decay extremely rapidly have no consequence to the evolution of the transient disturbance and may be ignored. We choose a decay rate of -3 as the cutoff and find disturbances that are linear combinations of eigenfunctions with slower decay rate.

The computations were performed in MATLAB. Our code uses a differentiation suite for the Chebyshev grid developed by Weideman and Reddy [36]. The objective functional was maximized by using the MATLAB generic nonlinear constrained optimization package FMINCON. The code has been validated by confirming the eigenspectra for the nonrotating channel flow at $R e=2000$ up to eight significant digits (Appendix A.7 in Ref. [30]).

\section{B. Stability boundaries}

The stability of a flow to small perturbations may be determined by either modal analysis or from the point of growth of perturbation energy. As mentioned earlier, the asymptotic stability of the flow is determined by the spectrum of the linearized operator. If the energy of any small disturbance decays monotonically for all time, then the flow is considered to be stable from an energy point of view. A flow which is asymptotically stable but not energy stable may display a transiently growing feature, which, but for nonlinear effects, will eventually decay [37]. This growth of disturbances can sometimes be sufficient to trigger nonlinearities, in which case the flow need not return to the initial base state. In shear flows, typically there is a stark difference in the critical Reynolds numbers obtained by the two measures. This is due to the nonnormal nature of the linearized operator $\mathbf{L}$.

We denote the critical Reynolds number, below which no disturbance mode grows exponentially, as $\operatorname{Re}_{c r M}$ (where $M$ stands for modal), and the energy critical Reynolds number as $R e_{c r E}$, above which $G_{\max }$ first exceeds 1 . In Fig. 2, we show how these critical values vary with the Ro. It can be seen that $R e_{c r} M$ obtained by modal analysis is highly sensitive to the rotation rate. Lezius and Johnston [10] first accurately derived the modal neutral curve by noting the analogy of the present 


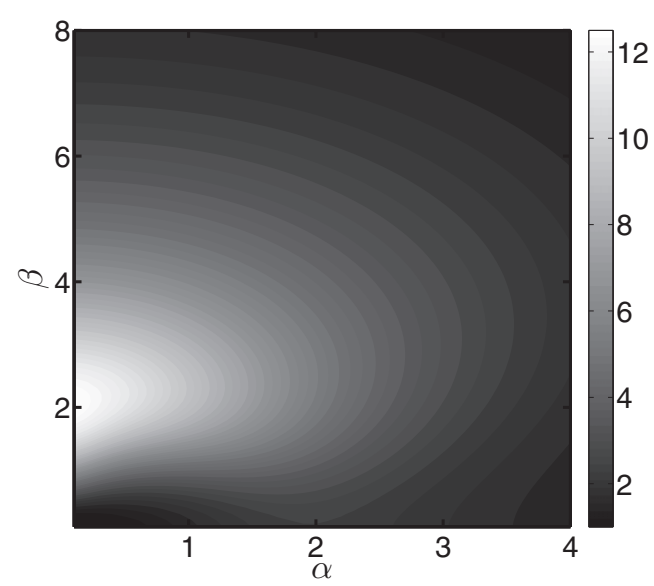

(a) $R_{0}=0.001$

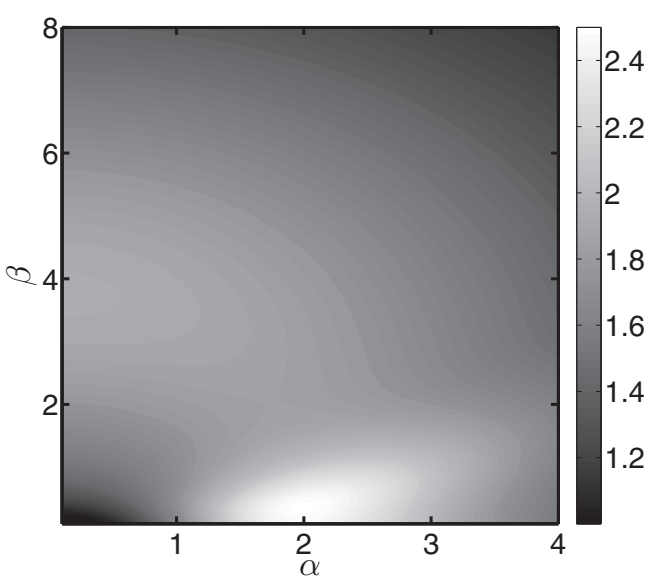

(b) $R o=0.8$

FIG. 3. The $G_{\max }$ contours for $R e=250$ for different $R o$. The optimal parameters are (a) $T_{\text {opt }}=18.72$, $\alpha_{\mathrm{opt}}=0.0, \beta_{\mathrm{opt}}=2.05, G_{\mathrm{max}}=12.64$; (b) $T_{\mathrm{opt}}=4.85, \alpha_{\mathrm{opt}}=2.00, \beta_{\mathrm{opt}}=0.36, G_{\mathrm{max}}=2.52$.

problem to the flow between two rotating cylinders in the narrow gap limit. The mode corresponding to the marginal state is seen to be streamwise independent. When the rotation rate is low, the spanwise wavenumber of the critical mode is around 2. As the rotation rate is increased, the corresponding critical mode has larger spanwise wavenumbers.

From Fig. 2, we see that $\operatorname{Re}_{c r E}$ is far less sensitive to changes in the rotation rate; it changes from 51.43 to 41.16 over three decades of magnitude of $R o$. In configurations between the neutral curves obtained by the two methods, disturbances may grow in energy for some time by a linear mechanism. We shall discuss the role of algebraic disturbances in the region in parameter space above the energy critical Reynolds number. It is also to be noted that the energy critical Reynolds numbers obtained for low rotation rates are close to 49.60, a value obtained by Joseph and Carmi [38] for the nonrotating channel flow (also see Ref. [37]).

\section{Modally stable regime}

We next study the transient growth characteristics in different regions of the $R e$ - $R o$ parameter space where exponential instabilities are absent. In Fig. 3 we demonstrate by a typical example that transient growth is qualitatively different to the left and to the right of the neutral stability boundary. For a fixed $R e(=250)$, the figure shows contours of $G_{\max }$ for representative low and high rotation rates. The $R o=0.001$ case is not markedly different from the corresponding results for a stationary channel at this Reynolds number. There, too, the disturbances that yield the largest growths are streamwise invariant [39]. They form rolls that evolve into streaks as a consequence of the vortex tilting lift-up mechanism [40]. On the other hand, at $R o=0.8$, the optimal disturbances are almost aligned along streamwise direction with a very small dependence on the spanwise coordinate. This suggests that the Orr mechanism is likely to be the more dominant energy amplification mechanism [41]. It may be noticed that the maximum achievable algebraic growth is lower when compared to the situation at low Ro.

In Fig. 4, we show level curves of $G_{\max }$ in the $R e$-Ro plane (outside the linearly unstable region). Two findings are immediately apparent. As in the example above, transient growth levels are much smaller everywhere on the right of the neutral boundary as compared to a corresponding Reynolds number on the left. Second, on a given side of the neutral boundary, $G_{\max }$ depends primarily on $R e$ and is rather insensitive to changes in $R o$. We can gain insight into these observations by examining 


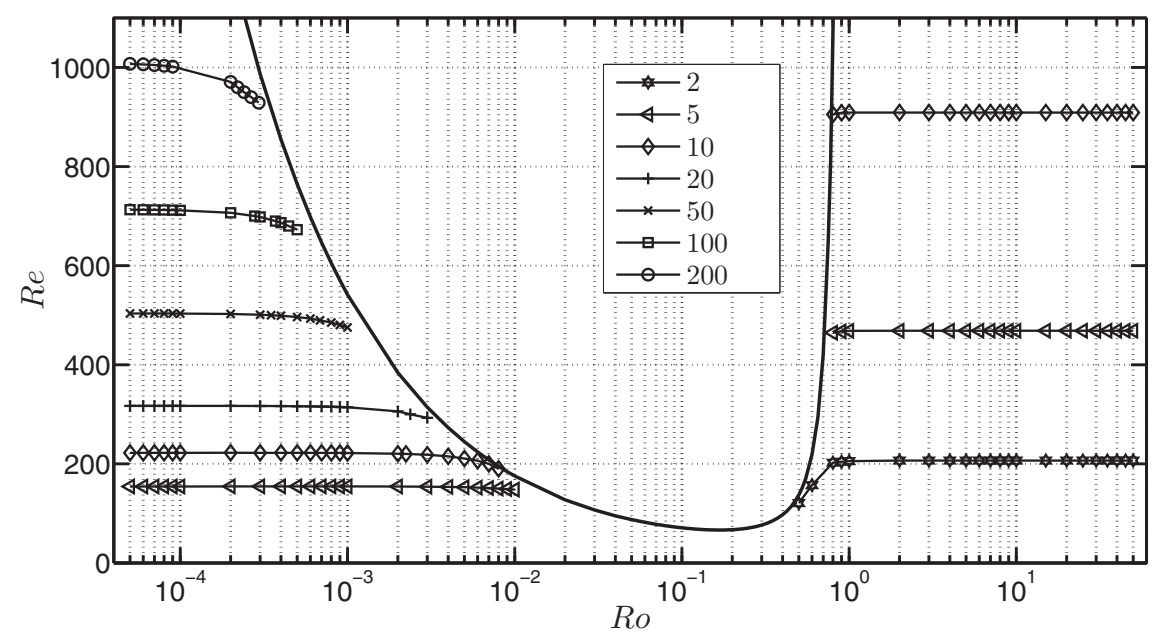

FIG. 4. Level curves of $G_{\max }$ are given. For low $R o$, the behavior resembles that of the optimal disturbances of the nonrotating channel. A large drop in the amplification levels is seen at high $R o$.

the different sources of nonnormality in the linearized equations [Eq. (7)]. The strength of the rotation then determines which of the sources of nonnormality will emerge stronger.

The major source of nonnormality is the forcing due to the normal velocity $\hat{v}$ in the normal vorticity equation, and it is this term that is responsible for the lift-up mechanism. An examination of the structure of the stability operator makes it evident that, at a given $\beta$, the departure from normality due to these operators decreases as the rotation rate increases. As in the nonrotating case, the largest amplifications are seen for disturbances that are streamwise independent at low rotation rates. For low rotation rates, the terms involving $R o$ serve to act as small corrections to the linearized operator. Hence, the growth in disturbance energies is similar to the growth seen in the nonrotating case. This translates to the lack of the dependence on $R o$ of $G_{\max }$ in the low rotation regime in Fig. 4.

The other source of nonnormality in the linearized equations is that the Orr-Sommerfeld operator $L_{O S}$ itself is not self-adjoint. This gives rise to a much weaker transient growth in two dimensions, which is completely independent of the rotation rate. As the rotation rate is increased, consistent with Taylor-Proudman arguments, the motion of the fluid is restricted to the plane perpendicular to the rotation axis. Thus, while disturbances favoring the lift-up mechanism are suppressed strongly, the disturbances amplified by Orr mechanism can still be excited at higher rotation rates. Disturbances initially having spanwise variation rapidly evolve to become two-dimensional with no flow along the axis of rotation. Thus the optimal disturbances in this regime evolve transiently only due to the Orr mechanism; i.e., $\beta_{\mathrm{opt}}=0$. Evidence of the Orr mechanism leading to the largest amplifications can be seen in Fig. 4 at high $R o$, where the level curves become horizontal and thus display insensitivity to the rotation rate.

In Fig. 5, we have plotted the $G_{\max }$ for specific values of $R o$ in different rotation regimes as a function of $R e$. The values obtained at higher $R o$ are shown to be at times an order of magnitude lower than for a small $R o$ for a given $R e$. It is seen here as well that $G_{\max }$ does not vary too much as the $R o$ is varied in different rotation regimes for large ranges of $R e$. At low rotation rates, as long as $R e$ is not sufficiently close to the critical value at given $R o$, the energy amplification obtained is found to obey the scaling laws due to Gustavsson's results [42] as the different curves coincide. It can be seen that for $R o=2.5 \times 10^{-4}$, as we increase $R e$, deviations from the $R o=0$ curve start to appear. This is a result of the values of $R e$ approaching the neutral boundary. Thus, it would be of interest to examine the regions close to the stability boundary in more detail.

In the high-rotation-rate modally stable regime, very close to the stability boundary, it can be seen in Fig. 4 that the level curves rise very slightly with $R o$. This implies that the value of $R e$ which 


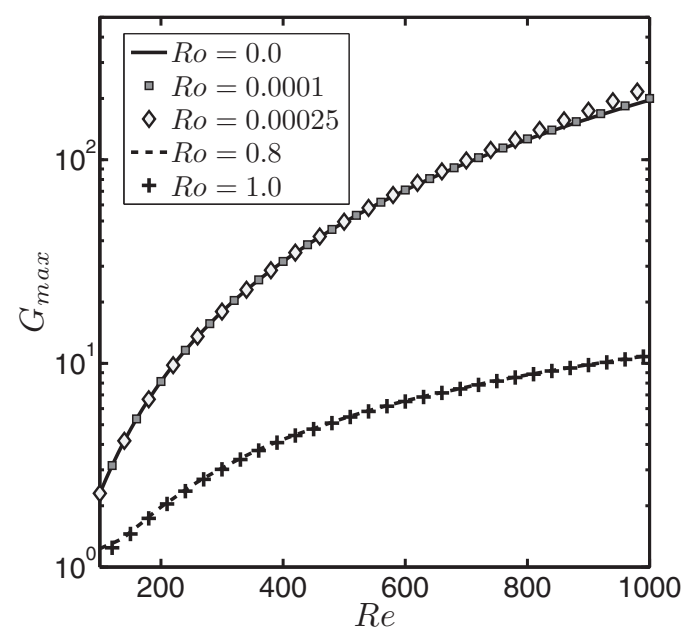

FIG. 5. A contrast between maximum transient energy growth at high and low rotation numbers.

yields a given energy growth increases with the rotation rate. It is important to note that if $\beta$ were to be identically zero, there would be no effect of the rotation on transient growth, since $R o$ would completely drop out of Eq. (7) ( $R o$ appears in the linearized equation only in the form $\beta R o$ ). The independence of the Orr mechanism on rotation was also demonstrated for the rotating Couette flow in the thesis of Daly [43]. Thus, the level curves not being perfectly horizontal near the stability boundary implies an oblique optimal structure; i.e., $\beta_{\text {opt }} \neq 0$. As we move to a region in $R e-R o$ parameter space further away from the stability boundary, the optimal amplification corresponds to that of the disturbance best amplified by the Orr mechanism at a given $R e$ as the spanwise variation is suppressed, consistent with Taylor-Proudman theory.

As we approach the neutral stability curve from the low $R o$ side, we see in Fig. 4 that the level curves of $G_{\max }$ noticeably dip toward a lower $R e$. This means that for a fixed $R e$, we have an increase in $G_{\max }$ as we approach the stability boundary. The typical behavior of optimal growth with $R o$ (for $R e=1000$ ) is shown in Fig. 6. The corresponding time at which this optimal growth is attained is also shown. The first modes that go linearly unstable are streamwise independent. The least stable modes have smaller decay rates as we approach the neutral boundary from the left, and hence the time before the modes individually decay is slightly longer. This allows for the lift-up effect to persist for a slightly longer time.

As mentioned earlier, the optimal structures obtained at low rotation rates are streamwise rolls that develop into streaks. This is similar to the nonrotating case in the sense that streaks are formed. However, due to the additional Coriolis force, the streaks are not symmetric about the centerline, with one side of the channel displaying a stronger streak than the other. This feature is more pronounced close to the stability boundary. Figure 7 shows the optimal structure for a typical low rotation $(R o=0.0002)$ and compares this to the nonrotating case. The velocities in the two cases are comparable.

The optimal disturbance is given in Fig. 8 for $R e=1000$ and $R o=0.8$. We can see that the rotation does not bias the occurrence of a secondary disturbance velocity toward any particular wall despite the high rotation rates. The strong rotation does little to alter the features of a flow that is largely confined to the plane normal to the rotational axis, i.e., the $x-y$ plane. Hence, disturbances may evolve by the Orr mechanism unhindered by the rotation.

\section{Modally unstable regime}

If there exists an exponentially growing linear mode for a given wavenumber vector, then an optimal analysis to determine the maximum amplification attainable over all time would not yield a 


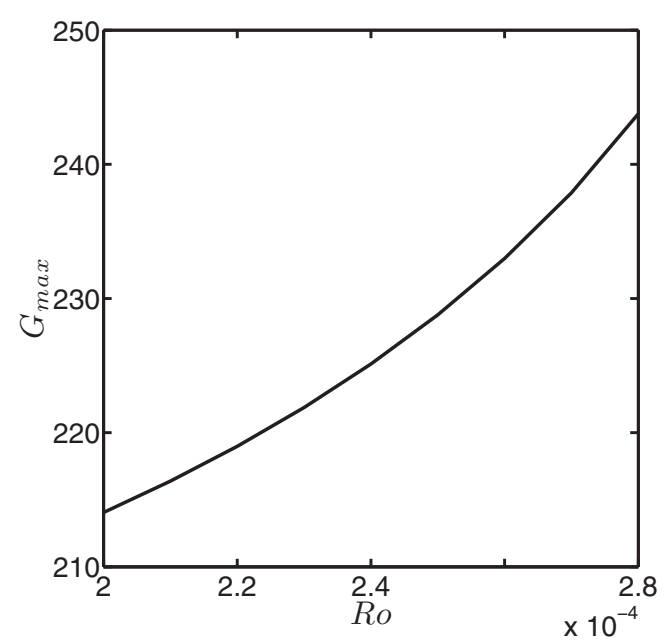

(a) $G_{\max }$ vs $R o$

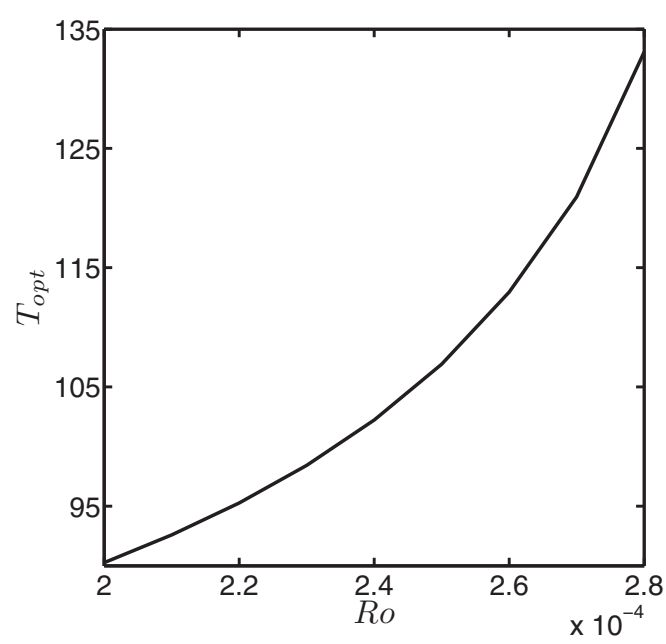

(b) $T_{\text {opt }}$ vs Ro 1000 .

FIG. 6. Optimal growth $G_{\max }$ and optimal time $T_{\text {opt }}$ when the neutral boundary is approached with $R e=$

finite value. Does this mean transient growth is inconsequential here? That remains to be verified. For the nonrotating channel flow, it was shown that algebraic growth can raise the energy of the disturbance before exponential instability definitively takes over the dynamics [39].

As the parameter space to be explored is vast, we shall limit our analysis to $R e=1500$ and some typical values of $R o$ where unstable modes exist; we have verified that our findings below are valid over a range of $R e$. The same set of pairs of $R e$ and $R o$ have also been used for nonlinear analysis using direct numerical simulations; the results will be presented in the next section. Hence, the discussion presented in this subsection will provide the necessary context for the results from the nonlinear simulations.

\section{Modal instabilities}

As we are considering parameters within the unstable regime, it would be instructive to see what region in wavenumber space modal instabilities exist. In Fig. 9(a), the curves represent the neutral

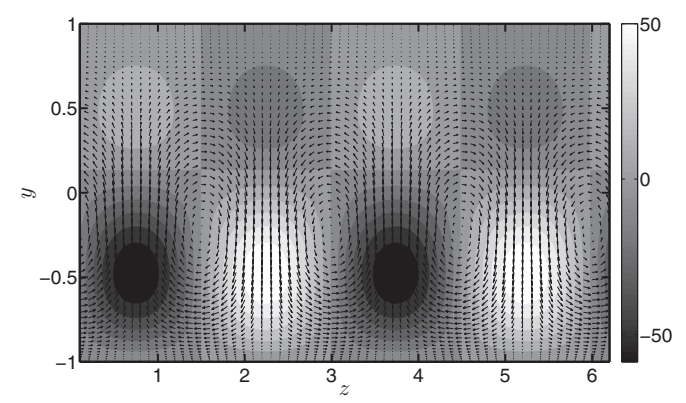

(a) $R o=0.0002$

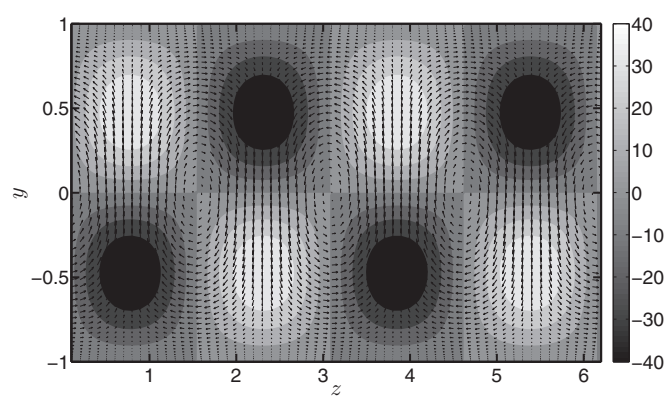

(b) $R o=0$

FIG. 7. The optimal structures for $R e=1000$ for low $R o$ (a), and for the nonrotating case (b), at the optimal times. The contours depict the magnitude of the streamwise velocity component. The velocity field $(v, w)$ are expressed through the vectors. The optimal parameters are (a) $T_{\mathrm{opt}}=90.27, \alpha_{\mathrm{opt}}=0, \beta_{\mathrm{opt}}=2.10$, $G_{\max }=214.05 ;$ (b) $T_{\text {opt }}=75.68, \alpha_{\text {opt }}=0, \beta_{\text {opt }}=2.04, G_{\max }=196.17$. 

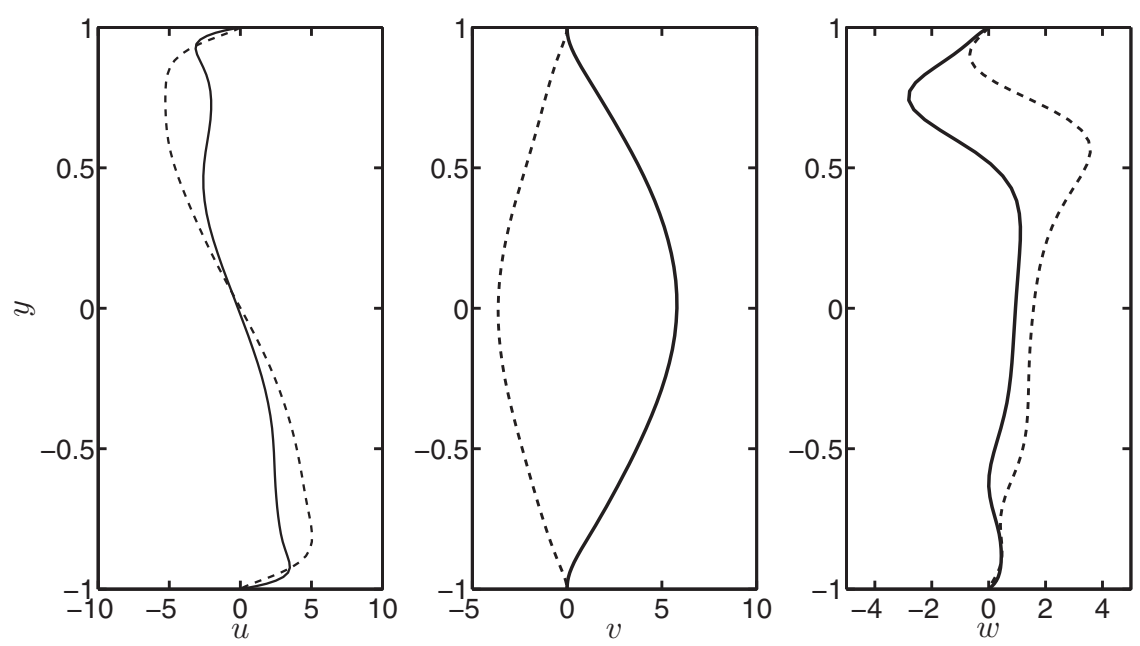

FIG. 8. The velocity components of the optimal disturbance at the optimal time when $R o=0.8$ and $R e=1000$. The real and the imaginary parts are given by the solid line and the dashed line respectively. The optimal parameters are $T_{\mathrm{opt}}=8.52, \alpha_{\mathrm{opt}}=1.75, \beta_{\mathrm{opt}}=0.169$, and $G_{\max }=11.03$.

boundaries in the $\alpha-\beta$ plane for different $R o$ when $R e=1500$. The wavenumber space bounded by each curve and the $\alpha=0$ axis are the modally unstable regions for the given rotation rate. Thus, the introduction of rotation brings in a large class of instabilities that was absent in the case of the nonrotating channel flow.

The largest growth rates correspond to modes that are streamwise independent. In Fig. 9(b), we show the exponential growth rate of the least stable streamwise-independent mode as a function of the spanwise wavenumber for different rotation rates; the growth rate is given by the real part of the eigenvalue $\left(\lambda_{r}\right)$. The growth rate associated with these unstable modes are large when compared to that of the Tollmien-Schlichting mode in the nonrotating channel. The spanwise wavenumber corresponding to the most unstable of these streamwise-independent modes does not remain constant; at larger rotation rates, the spanwise wavenumber of the most unstable mode increases. The observed trend is consistent with what is observed for the marginally stable modes along the neutral curve as the rotation rate is increased.

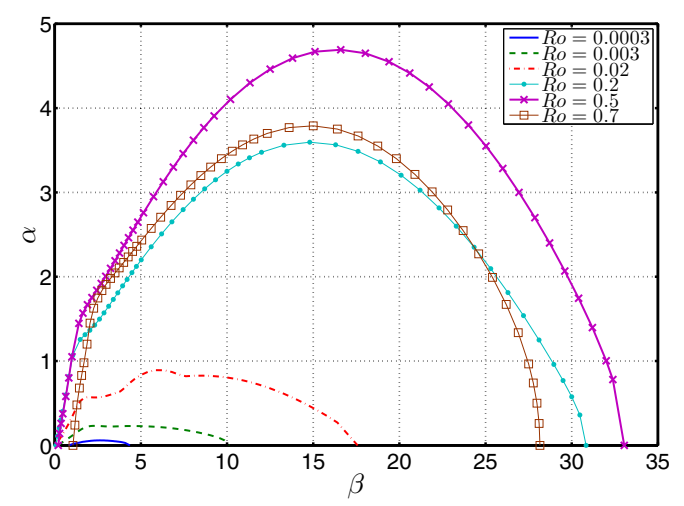

(a)

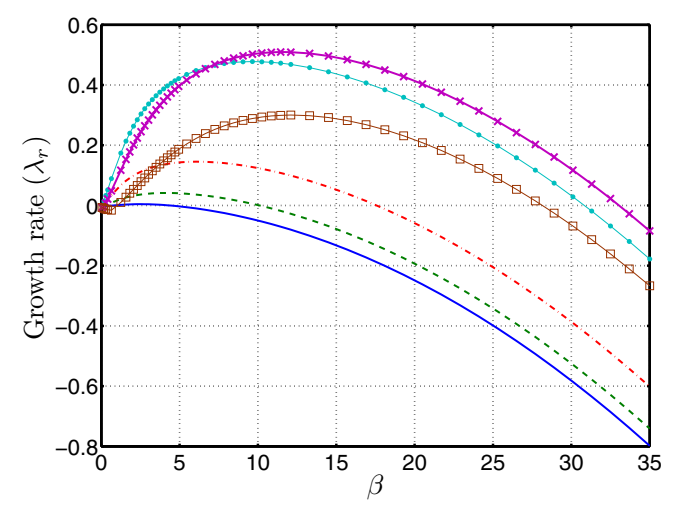

(b)

FIG. 9. $R e=1500$. (a) The marginal stability curves for various $R o$. (b) The exponential growth rate $\lambda_{r}$ of the least stable mode (with $\alpha=0$ ) as a function of $\beta$ for various $R o$. 


\section{Transient analysis}

We turn our attention back to the issue of characterizing algebraic growth mechanisms. As we are dealing with cases within the linearly unstable regime, we have to examine the extent of the role played by the unstable mode during the evolution of the disturbance. Now for a given rotation rate, we shall denote the most unstable eigenmode as $\hat{\boldsymbol{q}}_{u}$. We shall analyze the evolution of the initial perturbation for different rotation rates in the linearized setting governed by Eq. (7). $\hat{\boldsymbol{q}}(t)$ denotes the disturbance state vector at different times during the linear evolution of the perturbation. At this juncture, for every time, we define a vector $\hat{\boldsymbol{p}}(t)$ that is obtained by normalizing $\hat{\boldsymbol{q}}(t)$ to have unit kinetic energy as per Eq. (12). Thus, for $\hat{\boldsymbol{p}}(t)$, we simply have

$$
\hat{\boldsymbol{p}}(t)=\frac{\hat{\boldsymbol{q}}(t)}{\|\hat{\boldsymbol{q}}(t)\|_{E}} .
$$

To see if $\hat{\boldsymbol{q}}(t)$ is indeed coincident with the unstable mode $\hat{\boldsymbol{q}}_{u}$, we now take advantage of Eq. (12) and define a new quantity $M$ as

$$
M(t)=\frac{1}{2 k^{2}} \int_{-1}^{1} \hat{\boldsymbol{p}}^{H}(t)\left[\begin{array}{cc}
k^{2}-D^{2} & 0 \\
0 & 1
\end{array}\right] \hat{\boldsymbol{q}}_{u} d y .
$$

We can interpret $M$ simply as a measure of the projection of the disturbance onto the unstable eigenmode. When $M=1$, the disturbance has evolved such that it exactly coincides with the unstable eigenmode. It follows that $M$ will remain at 1 for all subsequent times after this point while the system behaves linearly.

We have already noted the limitations of doing a linear optimal perturbation analysis within unstable regime. However, our aim is to maximize growth at early times, so by specifying a target time $T_{\text {tar }}$, we can find the best possible initial condition with a particular wavenumber that maximizes the gain [given by Eq. (12)] obtained at that particular time; we denote this gain as $G_{\mathrm{opt}, \mathrm{t}}$. On obtaining the optimal initial conditions, we can find the extent of coincidence between the disturbance and the unstable mode at the initial and optimal times using Eq. (15). In addition to fixing $R e$ at 1500 , we shall also restrict the subsequent discussion to results for disturbances with wavenumber vector $\boldsymbol{k}=(0,2)$. The results do not qualitatively change on varying $R e$ and the disturbance wavenumbers within the modally unstable regime, and do not have a bearing on the overall conclusions.

In Fig. 10, we have plotted the maximum gain $G_{\mathrm{opt}, \mathrm{t}}$ obtained and the corresponding projection measures at different rotation rates for a range of values of $T_{\mathrm{tar}}$. For a comparison, we also plot the gain had the initial condition been the unstable mode; this gain is given by $G_{\text {uns,t }}$. We see that the maximum gain due to introduction of the optimal disturbances is greater than the corresponding gain seen when the unstable mode is used as the initial condition for all choices of the target time. In other words, if the initial conditions are the optimal ones, we get an initial spurt in disturbance kinetic energy followed by exponential growth, so the total disturbance kinetic energy is greater with algebraic growth than without. It is also telling that the unstable mode is never seen to be the optimal initial condition. This is a direct consequence of the nonnormal nature of the linearized operator. In the evolution of these optimals, the initial evolution is algebraic and this elevates the energy of the unstable mode that will continue evolving as per its associated growth rate after the other modes die out.

At this point, we can define a quantity $G_{\mathrm{a} / \mathrm{e}}$ as follows:

$$
G_{\mathrm{a} / \mathrm{e}}(t)=\frac{G_{\mathrm{opt}, \mathrm{t}}}{G_{\mathrm{uns}, \mathrm{t}}}
$$

Going back to Fig. 10, we see that beyond a certain target time ( $\left.\tau^{*}\right)$, the $G_{\mathrm{opt}, \mathrm{t}}$ and $G_{\mathrm{uns,t}}$ are directly proportional to each other. This suggests that the ratio $G_{\mathrm{a} / \mathrm{e}}$ is a constant $\left(\mathcal{G}^{*}\right)$ for all target times larger than this threshold value. This "saturated" ratio can serve as a measure of the nonmodal growth as it indicates the extent to which the energy of the unstable mode is enhanced by initial algebraic growth. 
JOSE, KUZHIMPARAMPIL, PIER, AND GOVINDARAJAN

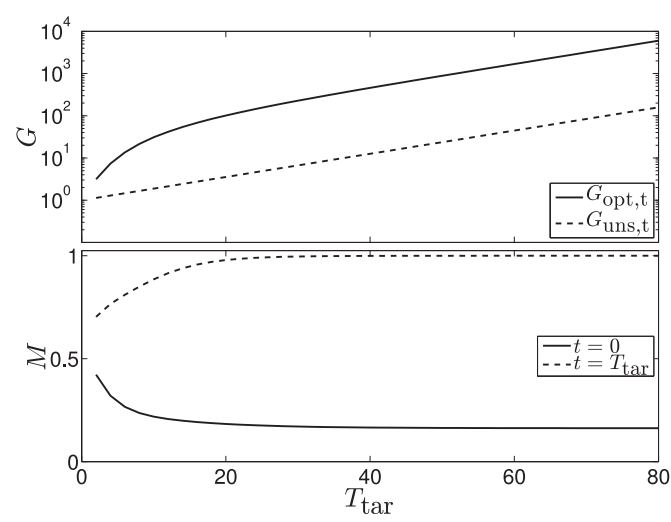

(a) $R o=0.003$

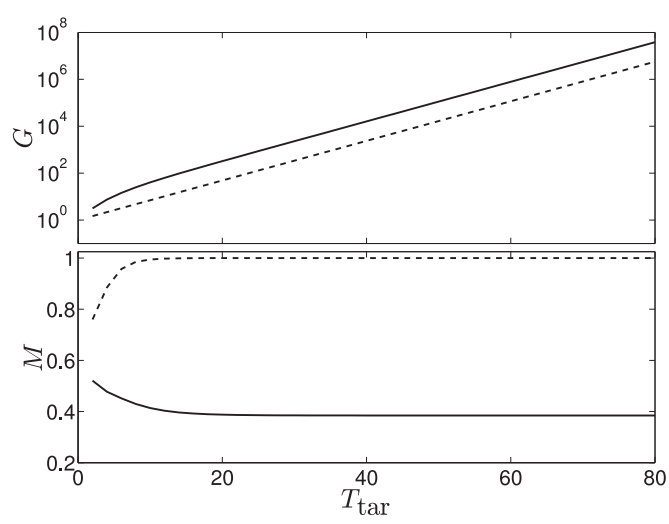

(b) $R_{o}=0.02$

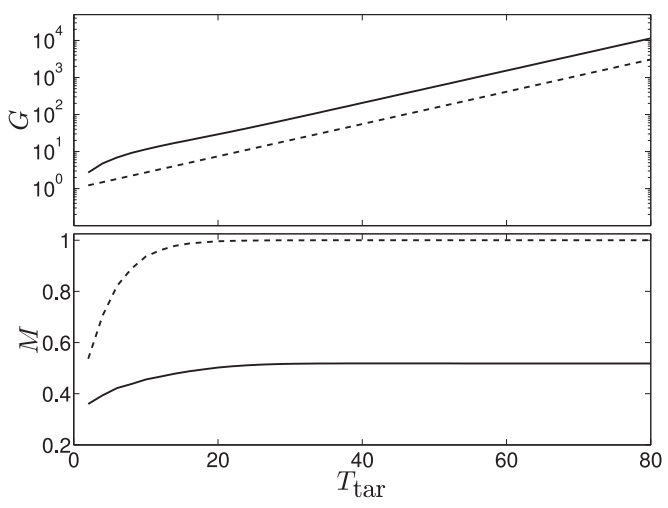

(c) $R o=0.02$

FIG. 10. The gains $G_{\text {opt,t }}$ and $G_{\text {uns,t }}$, and the projection measures $M$ when $T_{\text {tar }}$ is varied for different rotation rates. $R e=1500, \alpha=0$, and $\beta=2$.

We plot $\mathcal{G}^{*}$ and $\tau^{*}$ as a function of the rotation number $R o$ in Fig. 11. The values of $\mathcal{G}^{*}$ cover a wide range and are seen to be nonmonotonic in $R o$. The lowest values of $\mathcal{G}^{*}[\sim O(1)]$ are obtained when we are well away from the neutral stability boundary on both the low and high rotation portion

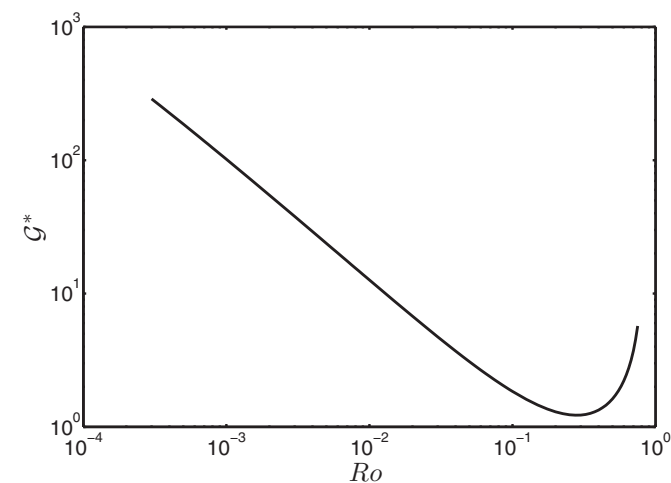

(a)

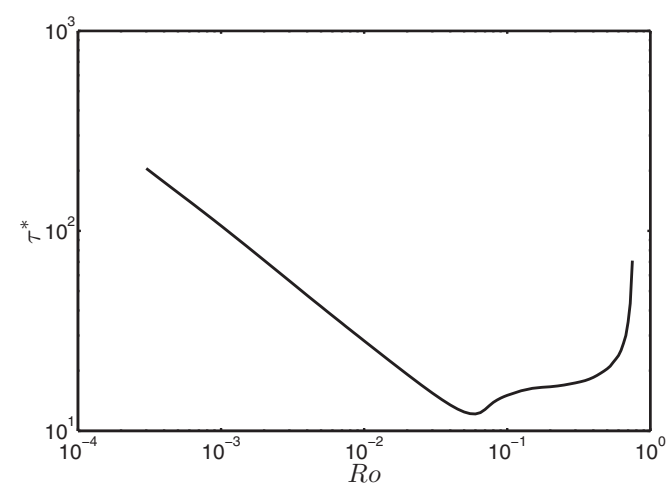

(b)

FIG. 11. (a) $\mathcal{G}^{*}$ and (b) $\tau^{*}$ at different $\operatorname{Ro} . \operatorname{Re}=1500, \alpha=0$, and $\beta=2$. 


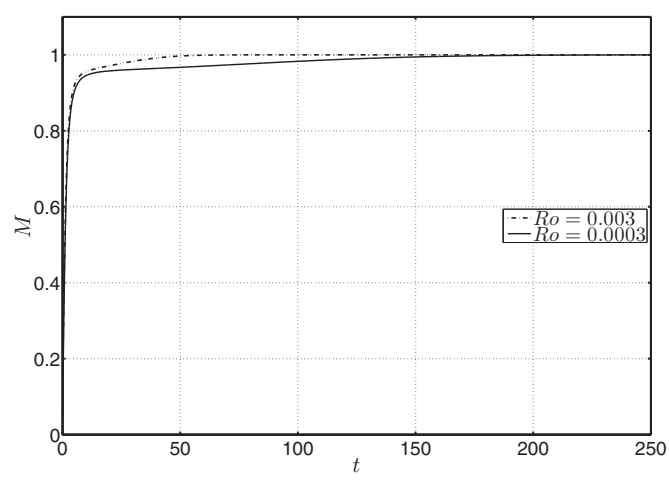

(a) Low rotation rates

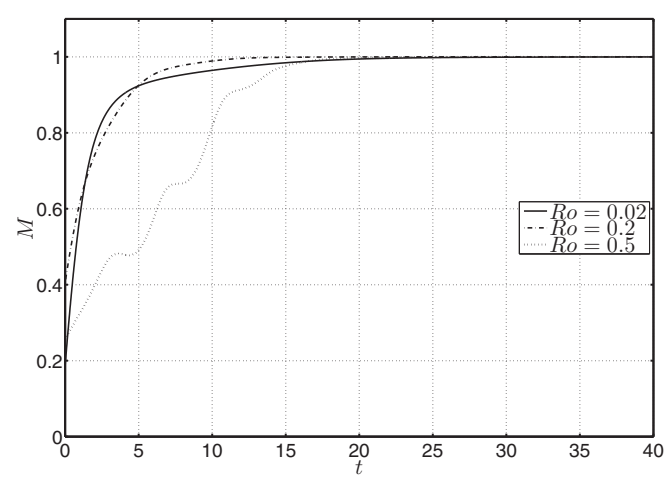

(b) High rotation rates

FIG. 12. The evolution of the projection measure $M$ defined in Eq. (15) for different rotation rates. Re $=1500, \alpha=0$, and $\beta=2$.

of the $R e-R o$ phase space. This is consistent with the fact that exponential instabilities in this range of $R o$ are readily excited. On the other hand, there is also a significant window in the low rotation range (nearly two decades of values of $R o$ ), where $G_{\mathrm{opt,t}}$ and $G_{\text {uns,t }}$ differ by well over an order of magnitude.

We estimate by $\tau^{*}$ the time at which the disturbance evolution is taken over by the unstable mode, which then drives the dynamics entirely. When the rotation rate is increased, i.e., when we go further into the linearly unstable regime, the time period before the unstable mode takes over and the dynamics becomes shorter. As our interest is in the relevance of algebraic growth, we see a range of $R o$ for which $\tau^{*}$ is not too small. This essentially suggests a window for the different nonnormal modes to interact with each other to produce a transient phenomenon. From the trends observed, for this set of rotation rates, there is a strong suggestion that nonmodal mechanisms are capable of playing a role in the transition process.

Thus far, the discussion involved finding an optimal perturbation for each value of the rotation rate while specifying a target time. We can also compare the extent to which the algebraic growth is prevalent at different $R o$ by fixing the initial condition. A suitable initial condition for the present study would be one that would evolve transiently in the absence of rotation. Once again with $R e=1500$ and $\boldsymbol{k}=(0,2)$, we choose the optimal perturbation when $R o=1 \times 10^{-4}$ as our initial condition; $R o=1 \times 10^{-4}$ belongs the modally stable region of $R e-R o$ phase plane, and therefore there is no trouble evaluating the optimal perturbation over all time. The method used here ties in with the conditions under which the nonlinear simulations discussed in the next section were performed.

We will start by examining when the values of $R o$ are small. So now we examine the projection measure $M$ defined by Eq. (15) for the two rotation rates that fall in the linearly unstable regime. We see in Fig. 12(a) at later times $M=1$, indicating that the disturbance has come to comprise the unstable mode alone. During the early stages of the evolution, the secondary flow evolves in a manner to form streaks in the flow as is in the nonrotating channel flow. At higher rotation rates, the unstable mode is excited relatively quickly as can be surmised from Fig. 12(b), where the projection measure $M$ [Eq. (15)] is plotted. Therefore, at higher rotation rates, the initial condition serves as a background out of which the unstable mode emerges.

The measures of $\mathcal{G}^{*}, \tau^{*}$, and $M$ calculated in this section offer some preliminary insight what might transpire in the transition picture for different $R o$. For a more complete picture, we now turn our attention to results obtained from nonlinear simulations.

\section{NONLINEAR SIMULATIONS}

In cases where a modal perturbation grows exponentially, or where transient growth is large, a nonlinear study is imperative to understand the next stage of evolution. We carry this out by direct 
numerical simulations of the three-dimensional Navier-Stokes equations in this flow, to characterize the transition to a new (steady or unsteady) state of the channel flow at different rotation rates. As discussed above, the rotational channel flow is a well-studied problem from both numerical and experimental points of view. In these studies, typically transitions away from the parabolic profile are achieved by the introduction of noise at a sufficiently high level such that instabilities are triggered, and the flow is allowed to evolve nonlinearly $[18,44]$. These studies were interested in the linearly unstable regime. In the present, we discuss results from nonlinear simulations with a wide range of $R o$, encompassing both regimes that are modally stable and those that are unstable. To make the discussion simpler, we present results at a Reynolds number of 1500, deeming them to be typical of the simulations we have carried out at other Reynolds numbers.

\section{A. Methodology}

The simulations were performed using the SIMSON code developed by KTH Mechanics, Stockholm [45]. A pseudospectral method is employed with Fourier expansions in the streamwise and spanwise directions, and a Chebyshev discretization is employed in the normal direction. For the results to follow, the horizontal directions are discretized using 64 Fourier modes each, and 81 Chebyshev polynomials are used for discretizing the normal coordinate. A second-order Crank-Nicolson scheme was used to discretize the linear terms, and the nonlinear terms were discretized by a four-stage Runge-Kutta (RK3) scheme. Periodic boundary conditions are used in the streamwise and spanwise directions, and at the walls, no slip and no penetration are imposed. In addition, all the simulations are performed with the mass flux through the channel kept fixed.

For validating the code, we imposed the least stable eigenmode as the initial condition at a very low amplitude $\left(10^{-6}\right.$ times the centerline base velocity) and found that the disturbance growth rates agrees with those predicted by linear stability theory for both the rotating and the nonrotating channel flows. For the rotating case, where the flow has undergone transition, we also verified that the total shear stress (sum of viscous and Reynolds stresses) varies linearly with the normal coordinate. The code has also previously been employed for numerous studies with the plane channel flow geometry (see Chevalier et al. [45]).

Here we wish to specify initial conditions that are favorable for algebraic evolution of the secondary flow. When the rotation rates are low, a streamwise independent initial disturbance is likely to grow transiently. Within the unstable regime as well, it was shown in the previous section that there is scope for algebraic amplification before the unstable modes dominates. For all the results here we fix $R e=1500$, a value where subcritical transition was previously observed in the nonrotating case [46]. The main results to follow are obtained with the optimal disturbance with wavenumber vector $\boldsymbol{k}=(\alpha, \beta)=(0,2)$ for $R o=10^{-4}$ provided as the initial condition. Within the regime of linear instability, we continue to use these initial conditions.

The computational box employed for all the cases is $x_{l} \times y_{l} \times z_{l}=2 \pi \times 2 \times 2 \pi$. Since our interest here is largely on the period leading up to transition, this is sufficient. However, it is to be noted that when one is interested in finding secondary instabilities (of twisting and wavy types), a much larger extent in the streamwise direction is desired [18]. We have ascertained that the short-time evolution of the flow varies insignificantly upon changing the streamwise extent of the computational domain. The computational box measures two wavelengths of the perturbation in the spanwise coordinate.

The initial kinetic energy has an amplitude of $25 \times 10^{-6}$ per box of size 1 wavelength of the disturbance in both streamwise and spanwise directions. In case of a zero wavenumber in either direction, an arbitrary length is fixed in that direction to define the box. The initial amplitude chosen is the lowest that leads to transition in the nonrotating case, and this is in agreement with the threshold values obtained in previous studies [46]. For cases that fall within the linearly unstable regime, we can also start with lower initial perturbation energy.

Particular care must be taken when the initial perturbation is streamwise independent, since these perturbations, through the nonlinear term in the governing equation, would act to excite only higher 


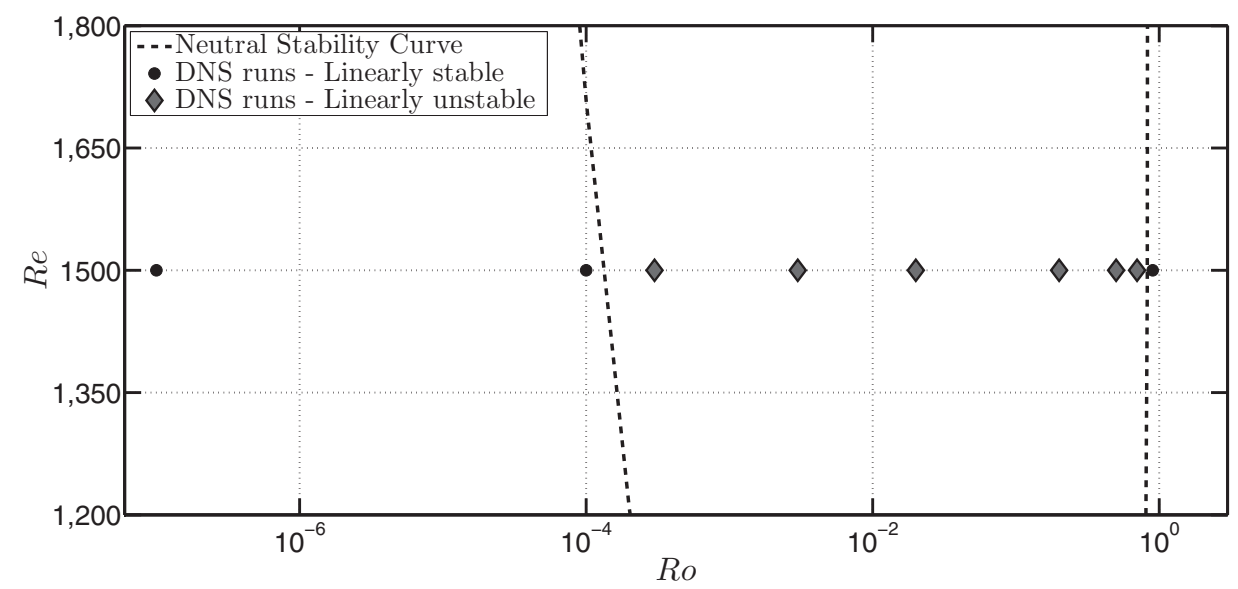

FIG. 13. The nonlinear simulations presented here are for the parameter values denoted by the symbols. $R e$ is fixed at 1500 .

harmonics of the initial spanwise wavenumber while remaining independent of the streamwise coordinate. In order to excite other streamwise spatial frequencies, we also introduce noise at a very low level at the start of the simulation $[30,46]$. The noise is introduced in the form of Stokes modes for a few nonzero streamwise wavenumbers. The total energy content of the noise is prescribed to be half a percent of that of the optimal perturbation, which ensures that noise is not the dominant factor in the dynamics and serves to trigger secondary instabilities. We have verified that the flow does not undergo transition to a new state when noise alone is introduced. The noise is added primarily to excite secondary instabilities.

The rotation rate is varied across some decades of $R o$ to clearly elucidate what happens in different regimes. In Fig. 13, we show the different cases considered and where they lie in the $R e-R o$ space with respect to the neutral stability boundary. The lowest $R o$ we choose is $1 \times 10^{-7}$, and this practically corresponds to the nonrotating case. On the low rotation side, we also study $R o=1 \times 10^{-4}$, which is just outside the stability boundary. Within the region where exponential instabilities may occur, we choose several points. The highest $R o$ we study is 0.9 , which does not yield exponentially growing disturbances at $R e=1500$; i.e., it lies to the right of the stability boundary. For ease of discussion, we shall refer to cases with $R o \leqslant 0.003$ as low rotation cases and to higher $R o$ as high rotation cases.

To get a measure of whether the transitioned flow is chaotic, we define an entropy $Q$ as follows:

$$
Q=\frac{1}{2 x_{l} z_{l}} \int_{\text {box }}\left[\omega_{x}(x, y, z, \tau+\Delta t)-\omega_{x}(x, y, z, \tau)\right]^{2} d \boldsymbol{x}
$$

where $x_{l}$ and $z_{l}$ are the streamwise and spanwise lengths of the box, and $2 x_{l} z_{l}$ is the volume of the periodic box. Streaks that have evolved from streamwise vortices have been seen as precursors to the transition process in several shear flows [47,48]. Hence, we define $Q$ based on the streamwise vorticity, as this gives us a picture of the fluid motion in the $y-z$. A similar approach had been employed by previously to quantify chaotic motion due to a flow past an inline oscillating cylinder [49]. On defining $Q$ by using other vorticity or velocity components, qualitatively similar results are obtained. We choose the reference time $\tau$ to be later than the time at which the initial transient behavior dies down. At $\Delta t=0$, we have $Q=0$. In a strictly periodic flow with period $T, Q$ will return to zero when $\Delta t=n T$, where $n$ is any positive integer.

In Table I, we have specified that the growth rates of the least stable eigenmodes with wavenumber vector $\boldsymbol{k}=(\alpha, \beta)=(0,2)$ when $R e=1500$ to serve as a reference. 
TABLE I. The exponential growth rates of the least stable mode with $\boldsymbol{k}=(\alpha, \beta)=(0,2)$ when $R e=1500$ at different rotation rates.

\begin{tabular}{lrlllr}
\hline \hline Ro & Growth rate & Ro & Growth rate & Ro & Growth rate \\
\hline $1 \times 10^{-7}$ & -0.00431158 & 0.003 & 0.03165619 & 0.5 & 0.19551277 \\
$1 \times 10^{-4}$ & -0.00087845 & 0.02 & 0.09718189 & 0.7 & 0.05017800 \\
$3 \times 10^{-4}$ & 0.00398466 & 0.2 & 0.25758712 & 0.9 & -0.01717999 \\
\hline \hline
\end{tabular}

\section{B. Nonlinear results: Low rotation rates}

To start off, we shall first consider the cases where the rotation rates are small. As a measure of transition, we examine the time evolution of the root mean square (rms) values of the different components of the velocity for all the cases. For all the cases, there is an initial period where there is transient amplification of the disturbance. Several wavenumbers then start to gain energy (not shown) through the nonlinear terms aided by the initial noise. The transient amplification seen at early times is then inhibited by nonlinear effects. For both low and high rotation cases, we seek to describe the flow characteristics well after the initial transients have run their course, and the resulting flow is fully nonlinear.

It can be seen in Fig. 14 that the rms values of the streamwise velocity $u$ settle within a range of amplitudes not very dependent on the rotation rate. A transient spurt in rms values of the various velocity components is followed by a settling down into a time-dependent state at a lower mean energy level than the maximum transient. In this state, the rms values show an apparently chaotic signal for all the cases with similar time-averaged behavior. The observations when other components of the velocity are considered offer similar trends. We emphasize that this is the case regardless of the fact that some of the configurations here $\left(R o=3 \times 10^{-4}, 0.003\right)$ can support exponential instabilities. We also show in Fig. 14(b) that the initial evolution of the secondary flow appears to be similar while the nonlinear terms have not fully come into play. Departures from this type of behavior are seen when the rotation rate is increased. This prepares us for pronouncedly different behavior at high rotation rates.

The rms values do not, however, tell us about where the secondary flow is set up and what the dominant structures are. The linear stability results lead us to expect that the Coriolis force biases the flow toward stronger secondary flow near the high-pressure side of the channel. In Fig. 15, we show vortex core regions identified by use of the $\lambda_{2}$ criterion [50]. $\lambda_{2}$ is the second eigenvalue of the tensor $\mathbf{S}^{2}+\mathbf{R}^{2}$, where $\mathbf{S}$ is the strain rate tensor and $\mathbf{R}$ is the antisymmetric part of the velocity

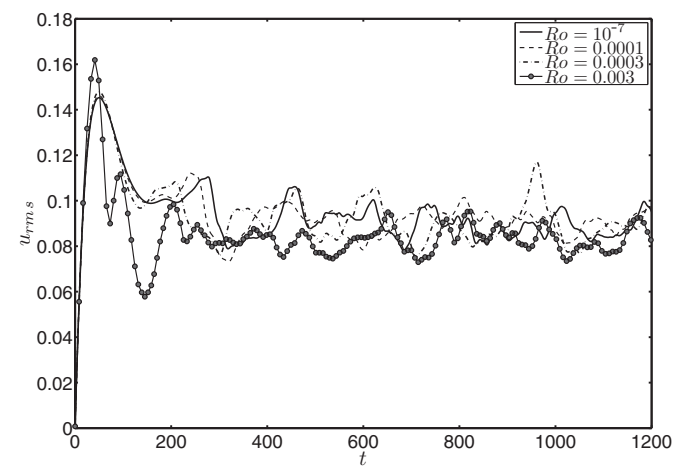

(a)

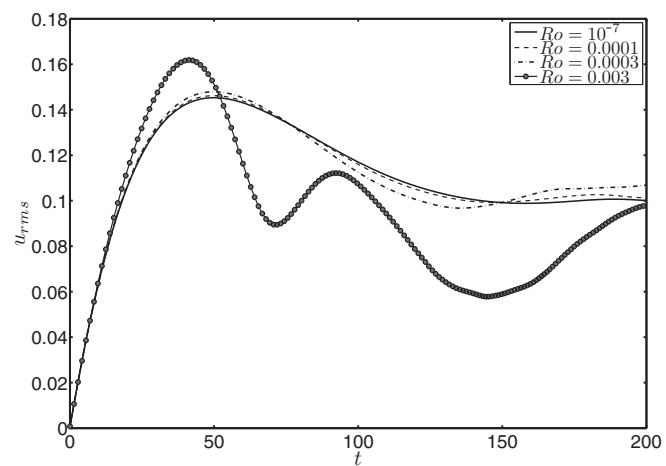

(b)

FIG. 14. (a) Evolution of root mean square (rms) values of the streamwise velocity component. (b) A magnified portion of panel (a) highlighting the early stages of the evolution of the secondary flow. 


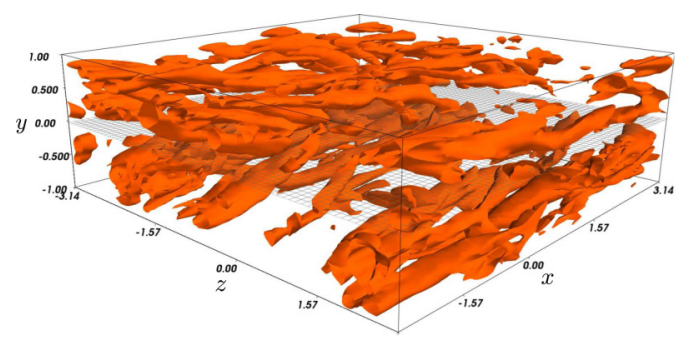

(a) $R o=10^{-7}$ (Subcritical)

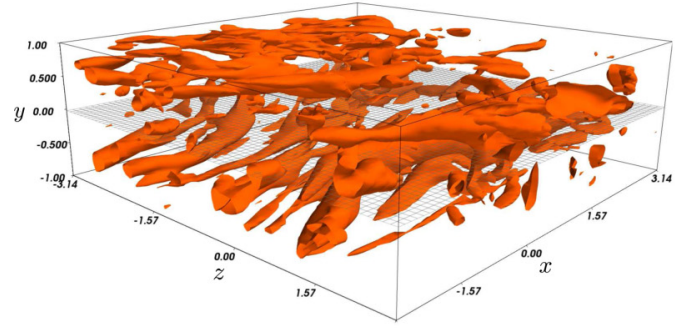

(c) $R o=3 \times 10^{-4}$ (Supercritical)

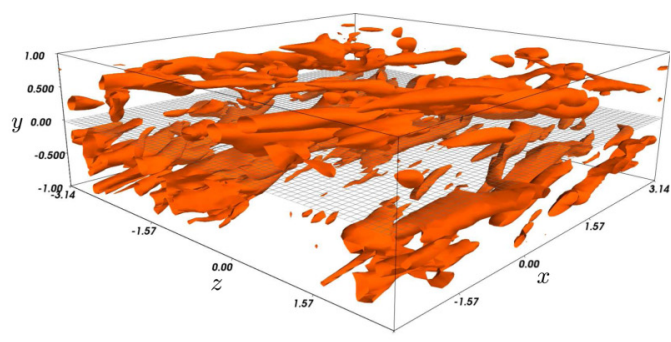

(b) $R o=10^{-4}$ (Subcritical)

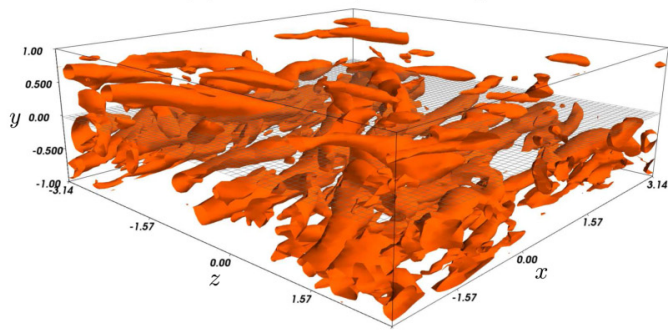

(d) $R o=3 \times 10^{-3}$ (Supercritical)

FIG. 15. The isocontours of constant $\lambda_{2}=-0.05$ at various rotation rates. The vortex cores are identified in each of the cases at $t=500$, a characteristic time after the initial transient behavior has died out.

gradient tensor (the rotation tensor multiplied by 0.5 ). We choose a characteristic time $t=500$ for comparing the different cases such that the initial transient behavior has run its course and the flow is fully nonlinear.

When the rotation rate is low, we see the vortex structures to be distributed across the channel and to be disordered. The behavior is qualitatively similar in all cases and similar to that in a stationary channel. At early times (not shown), the secondary flow initially gets set up as aligned in the streamwise direction and then breaks down to give a seemingly chaotic flow [46]. Thus, the flow at low $R o$, even if within the linearly unstable regime, can undergo transition via mechanisms by which subcritical transition occurs in the nonrotating channel flow. At this point, no clear indication of the role of the unstable mode is seen.

In Fig. 16 are shown mean flow profiles of the present simulation for different values of $R o$ considered. The mean flow is derived by averaging in the streamwise and spanwise coordinates and in time. The profiles obtained resemble that of nonrotating turbulent channel flow. It was seen in earlier work that the mean flow in the rotating channel is no longer symmetric about the centerline due to the Coriolis force when the flow becomes turbulent [22,23]. Despite being in the linearly unstable regime, here we do not see strong manifestations of the asymmetry in the mean flows despite the Coriolis force being capable of exciting instabilities in a couple of the cases.

The rms values in Fig. 14 suggest a strongly fluctuating velocity field. To get a sense of how chaotic each of the resulting flows are, we now turn to the entropy measure $Q$ defined in Eq. (17). Setting the reference time $\tau$ as 500, in Fig. 17, we plot the evolution of $Q$ for the different cases considered. The plots make it evident that we have chaotic flow, since in no case do we have $Q$ returning to zero. In addition, the range of values of $Q$ seen for the different cases is not drastically different.

Thus far, there has been no indication of whether the unstable mode plays a significant role during the transition process. The results from the linear analysis in the previous section suggest that the disturbance initially grows algebraically despite the presence of the unstable mode (for the unstable configurations considered here). The nonlinearity in the governing equations act to limit the linear growth once the secondary flow has become sufficiently strong. Then the peak of the rms values in Fig. 14 can be considered a marker for when the flow has become fully nonlinear; this is seen to 


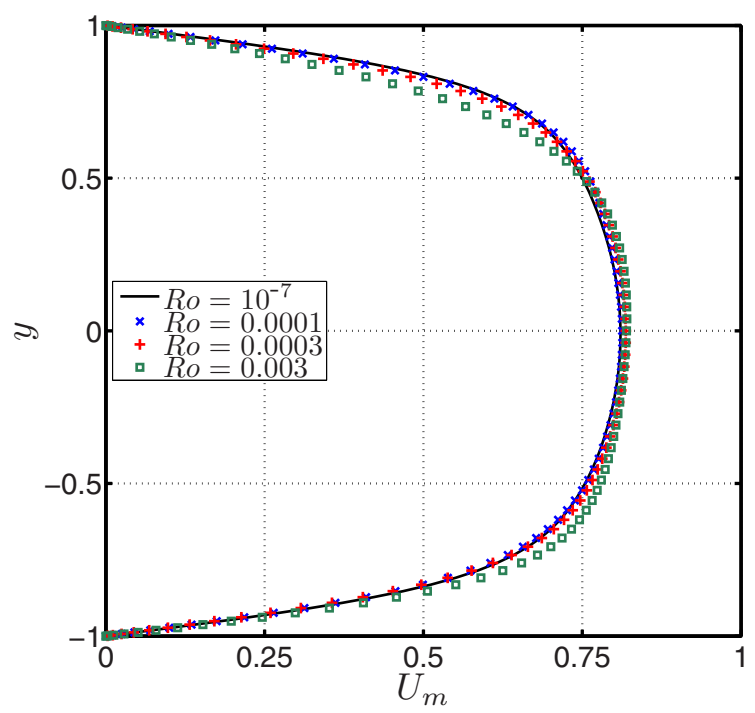

FIG. 16. The temporally and spatially averaged mean flow profiles for different rotation rates. The familiar profile of a turbulent channel flow is obtained for low rotation rates.

occur at $t \approx 50$ for all the cases. At the same stage in the linear evolution, the unstable mode has not yet come to dominate the dynamics [see Fig. 12(a)]. The algebraic evolution of the disturbance has been so strong such that the flow becomes fully nonlinear without the linear unstable mode having completely emerged.

In the case of the nonrotating channel flow, the optimal perturbation eventually decays if the nonlinear terms are not triggered. This happens when the energy content of the perturbation is initially very low, and the algebraic linear amplification is not strong enough to render the nonlinear terms important. If we were to introduce the perturbation at lower energy levels, the secondary flow can evolve linearly for long enough to coincide with the unstable mode. This is clearly depicted in Fig. 18 when $R o=3 \times 10^{-4}$. As we increase rotation such that we are further away from the

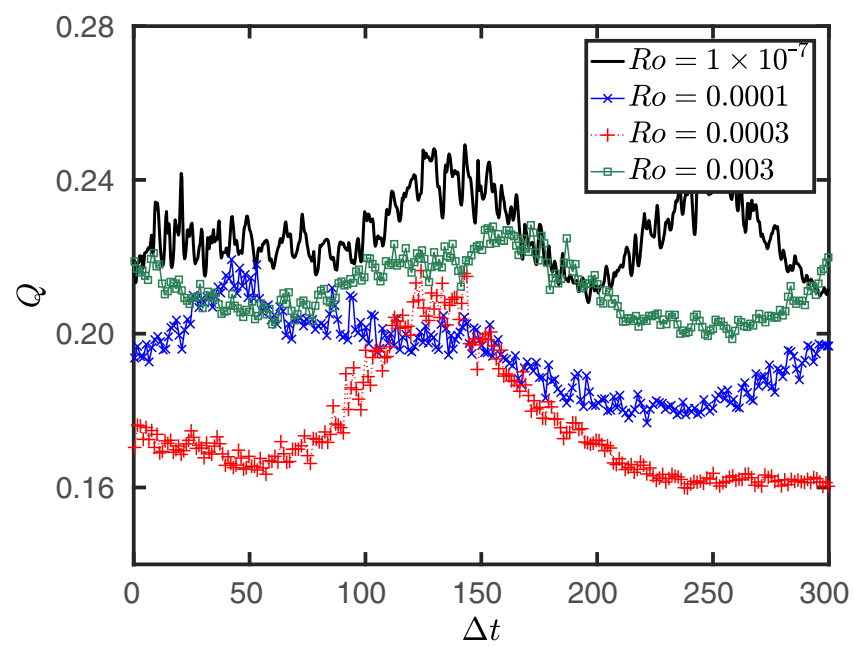

FIG. 17. The evolution for the measure of chaos $Q$ for different $R o$. For all the cases, a departure from the initial state is shown. Here we choose $\tau=500$ as the starting state. 


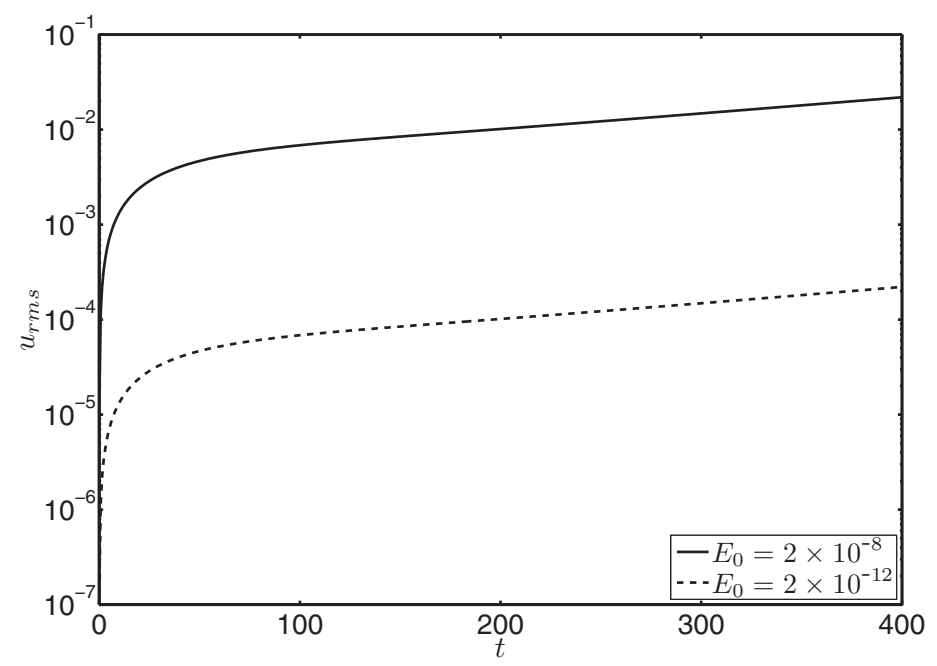

FIG. 18. The unstable mode is eventually excited in the full nonlinear setting when initial perturbation energy is low $\left(R o=3 \times 10^{-4}\right)$. Algebraic growth is seen at early times before the unstable mode becomes dominant.

stability boundary, the time taken for the unstable mode to emerge becomes shorter. Upon exciting the unstable mode, the flow would evolve as dictated by the exponential growth rate from linear theory until the nonlinear terms become important.

From Fig. 18, we also see that the initial rise in the rms values happen at a much faster rate than the growth rate specified by the unstable mode. Eventually the unstable mode alone is seen to dominate. At the stage where the unstable mode alone survives, the secondary flow is seen to have already attained a kinetic energy that is larger than what would have achieved had we started with an unstable mode alone. We can then say that given a class of initial conditions with the same kinetic energy, algebraic disturbances can enhance the energy content of the unstable mode and make the flow nonlinear at earlier times.

So in the cases considered above at low rotation rates, algebraically growing disturbances have been shown to be capable of triggering transition in two ways. First, the algebraic growth can be strong enough such that the nonlinear terms come into play. The transition is triggered by the vortex stretching and tilting mechanisms that lead to the formation of streaky structures. Alternatively, if we impose the initial energy content of the disturbance to be very low, we end up with the situation where the unstable mode is eventually excited and transition occurs by the secondary instabilities of the saturated flow. However, in contrast to noisy environments, the unstable mode emerges with much higher energy due to the algebraic amplification of the disturbance. With regard to that point when the evolution transition has occurred, the nonmodal mechanisms are more dominant at short times. However, if the flow has not undergone transition by subcritical mechanisms, the unstable mode will trigger the nonlinearity at later stages.

\section{Nonlinear results: Intermediate and higher rotation rates}

As the rotation is increased, we are now very much within the modally unstable regime. In the previous subsection, we already saw signs of the unstable mode emerging in the dynamics provided that the flow has not become fully nonlinear until a certain time. The range of rotation rates considered here are more in line with earlier studies where strong instabilities and transition to turbulence have been observed. The question then posed here would be to see if the nonnormal nature 


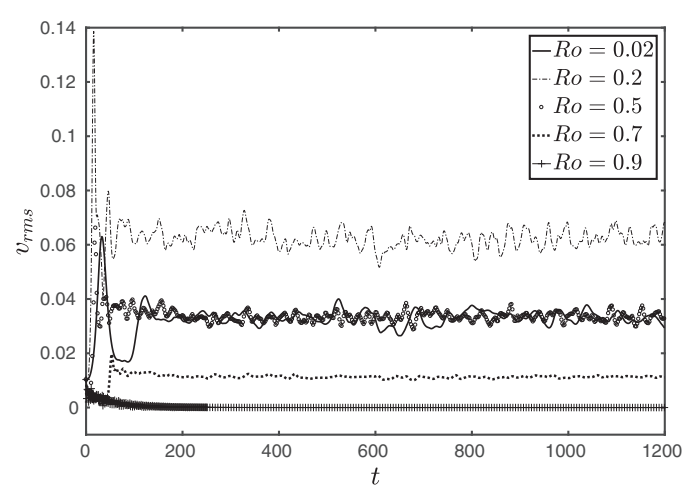

(a)

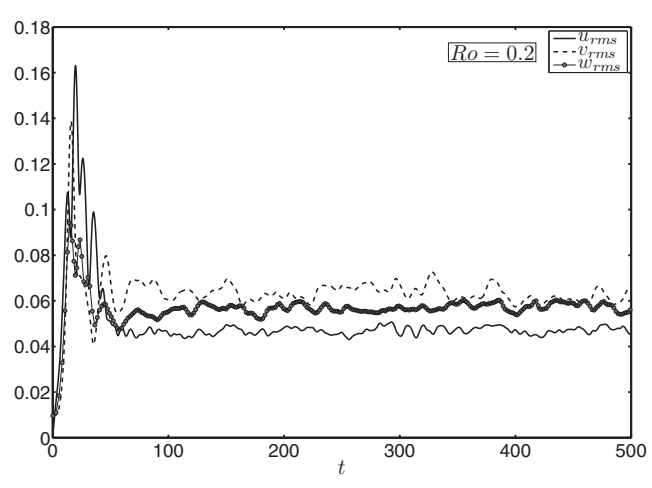

(b)

FIG. 19. (a) For the high rotation cases, the evolution of the root mean square (rms) values of the normal velocity component. (b) The rms values of the different velocity components when $R o=0.2$. The secondary flow becomes more isotropic when the rotation rate is increased.

of the governing equations and algebraic disturbances have any significant effect on the dynamics of the flow.

As before, we first examine the rms values of the resulting flow at various rotation rates in Fig. 19. A notable algebraic behavior, as was evident at lower values of Ro (Fig. 14), is not very apparent here. In Fig. 19(a), for certain cases ( $R o=0.2$, for instance), the rms value (perturbation energy) rises drastically in a short period, and consequently the flow rapidly becomes nonlinear. One must be remember that the exponential growth rates of modes for these values of $R o$ are fairly high, and the linear regime lasts for a very short window. For the values of $R o$ considered here, the results from the linear analysis also do not predict pronounced algebraic growth effects (see Subsec. III D).

The rms values of the streamwise velocity get suppressed to a greater extent (not shown). For the other velocity components, the rms behavior is not monotonic as we increase the rotation rate [see Fig. 19(a)]. What was initially a more streamwise dominant flow in the case of low rotation rate cases now has comparable rms values of velocity in all directions; the $R o=0.2$ case displays this the best [see Fig. 19(b)]. Inside the linearly unstable region, the rms values of the spanwise and normal velocity components increase in a range of $R o$. These values once again get suppressed as the rotation is further increased, and the region of linear stability is approached. Also to be noted is that on increasing the rotation rates, the rms values display smaller deviations from their long time average [for instance, compare the cases with $R o=0.2,0.7$ in Fig. 19(a)].

From Fig. 19(a), the flow has undergone a transition to an unsteady state at all rotation rates except $R o=0.9$. For this case, the high rotation rates effectively kills all the fluctuations very quickly, in keeping with Taylor-Proudman arguments. The resulting flow then quickly reverts back to the parabolic flow. For the parameters considered here, in the rapidly rotating case, both exponential and algebraic growths of perturbations are suppressed. One must keep in mind that when $R e>5772$, transition due to the breakdown of the two-dimensional TS waves, which is unaffected by rotation, is still possible [15].

When we consider the $\lambda_{2}$ structures in Fig. 20, they are found to be increasingly concentrated at the lower, high-pressure side of the channel. The structures appear to be far more disordered when the rotation rate is far from either linear stability boundary ( $R o=0.2$, for example). The vortex structures formed also appear to be more ordered along the streamwise coordinate as the rotation is further increased. The conspicuous absence of vortical structures on the low-pressure side of the channel suggests that the flow remains largely ordered and laminar in that region. Thus, the Coriolis force acts to effectively laminarize at least one side of the channel flow. Such behavior was seen in Ref. [10], where the authors found a reduction in the turbulent intensities near the low-pressure wall. As we increase the rotation rates further, the Coriolis force acts to the confine the secondary 


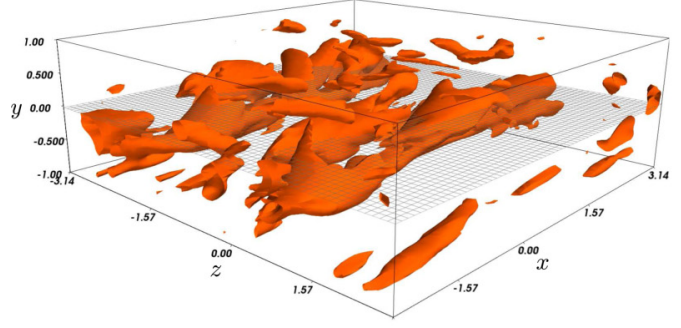

(a) $R o=0.02$

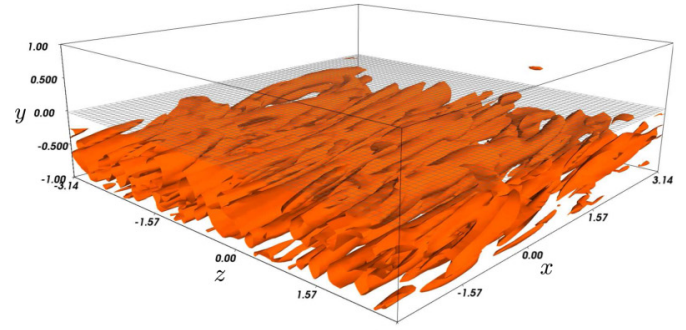

(c) $R o=0.5$

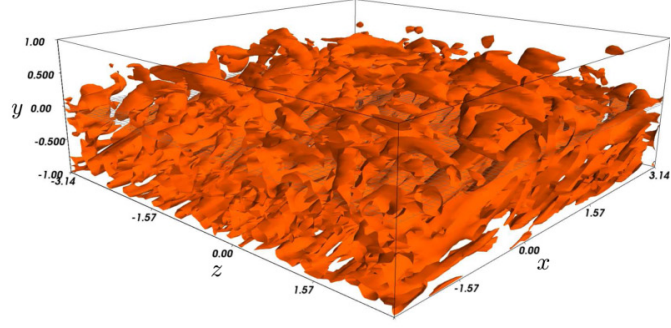

(b) $R o=0.2$

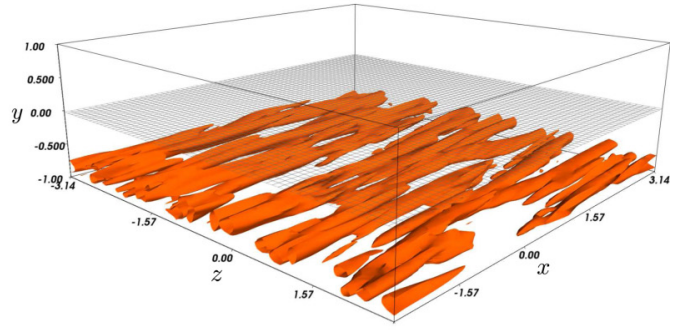

(d) $R o=0.7$

FIG. 20. The isocontours of constant $\lambda_{2}=-0.05$ at different rotation rates. All cases are supercritical. The vortex cores are identified at $t=500$, same as in Fig. 15.

motion to smaller regions in the channel. Thus, the secondary flow set up is much weaker, as can be seen for the $R o=0.7$ case in Fig. 20.

The mean flow obtained for these rotation rates are given in Fig. 21. The case of $R o=0.2$ displays clear departure from symmetry about the centerline. It is noticed that the velocity profile is linear over a significant portion of the channel width. This portion is the region where the strong vortical structures seen in Fig. 20 exist. A similar correspondence of linear velocity profiles and strong

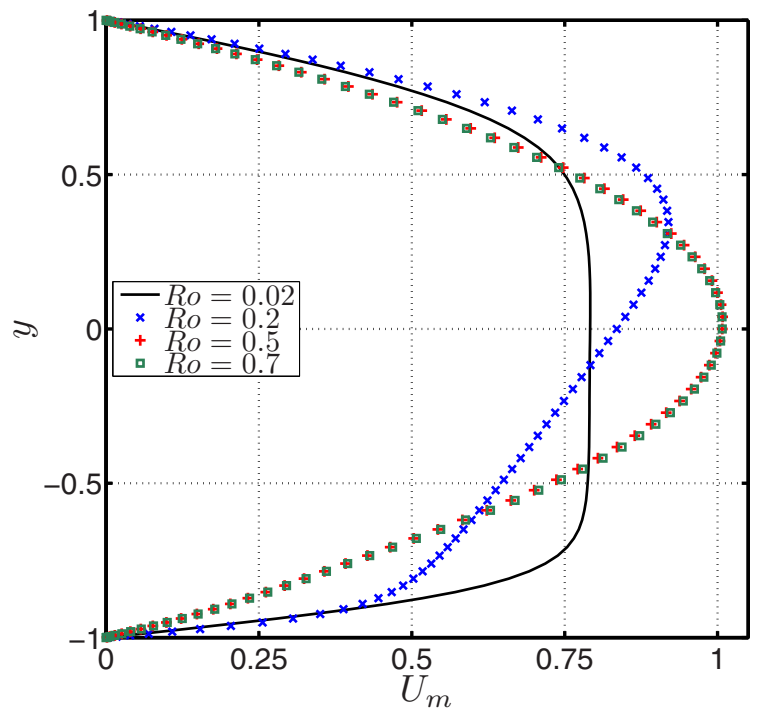

FIG. 21. The temporally and spatially averaged mean flow profiles for different rotation rates. Prominent asymmetry is seen at higher rotation rates. 


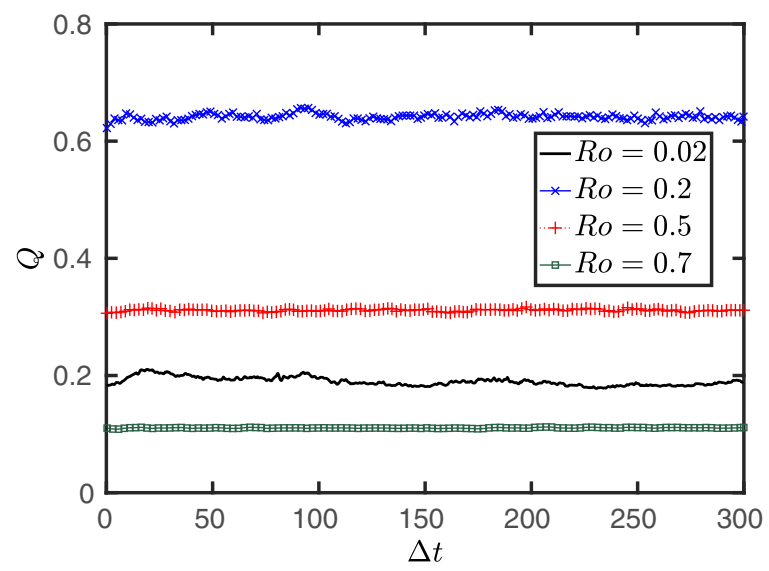

FIG. 22. The evolution for the measure of chaos $Q$ for different $R o$. For all the cases, a departure from the initial state is shown. Here we choose $\tau=500$ as the starting state.

structures has been reported earlier for turbulent rotating shear flows [23,51-53]. We comment here that the resulting mean flow is not different from the case if we induce transition using the unstable mode.

To get a picture of how chaotic the resulting flows are after transition, we plot entropy $Q$ in Fig. 22. It is seen that for none of the cases does $Q$ go to zero, and hence the resulting flows are chaotic. Additionally it must be noted that $Q$ defined in Eq. (17) is an integral measure over the computational box. At these rotations, we are seeing one side of the channel getting laminarized with the vortex structures concentrated on the other side. This means that the contribution to $Q$ in these cases is not very significant in the laminarized side of the channel. Despite such a situation, the values of $Q$ are fairly high when compared to the low rotation cases. This suggests that the regions where the secondary flow does persist offer extremely chaotic dynamics.

\section{CONCLUSION}

In this article, we focus on the role of algebraic disturbances in the transition scenario of the rotating channel in various rotation regimes. We show that the critical modal Reynolds number for the rotating channel flow does not coincide the energy critical Reynolds number for all rotation regimes. As a consequence, transient amplification of disturbances is observed in modally stable regions in the $R e-R o$ phase plane. Interestingly, we show that the energy critical Reynolds number is only feebly sensitive to the rotation rate, in stark contrast to the modal behavior. On a given side of the modally stable region (i.e., at low and high $\mathrm{Ro}$ ) at a given Reynolds number, the optimal transient growth does not vary much with the rotation rate at a given $R e$. However, the insensitivity to rotation on either side of the neutral curve has very different origins. It is only in the vicinity of the neutral boundary in $R e$-Ro parameter space that discernible changes in the optimal characteristics are observed.

At low rotation rates, the transient growth of disturbance kinetic energy is due to the lift-up effect and is subsequently shown to be important while considering transition. The optimal transient growth amplitudes and the corresponding optimal wavenumbers obtained are close to those for the nonrotating channel. It is a consequence of rotation serving as a correction to the dynamics of the channel flow. However, even at extremely low rotation rates, the optimal structure breaks centerline symmetry due to the Coriolis force, with larger asymmetry closer to the neutral boundary. At extremely large rotation rates, consistent with the Taylor-Proudman theorem, all variation along the axis of rotation is inhibited. The streamwise-independent disturbances which yield the largest transient growth at low Ro are therefore now suppressed. Thus, we see only weakly growing 
perturbations that evolve transiently due to the Orr mechanism that is completely unaffected by the strength of the rotation.

Within the modally unstable regime, we have shown that initial disturbances can grow algebraically before the most unstable mode alone emerges. While it is impractical to perform an optimization over all wavenumbers and time, we can deal with a more tractable problem by specifying a target time $T_{\text {tar }}$, where we are interested in the initial condition yielding the largest gain. Beyond a particular target time $\tau^{*}$, the initial condition that gives the best gain in comparison to when the unstable mode is alone introduced becomes invariant. This suggests $\tau^{*}$ defines a window where nonmodal behavior prevails before the unstable mode becomes dominant. For a wide range of values of $R o$ within the unstable regime, this algebraic gain suggests that linear nonmodal mechanisms are still important as they raise the energy of the unstable mode and accelerate the transition process.

We solve the incompressible Navier-Stokes equations using direct numerical simulations for better understanding the processes in the modally unstable regime. When the rotation rate is low, both modally stable and unstable, we have shown that transition occurs similarly to the subcritical manner of the nonrotating case. The transient amplification of the disturbance triggers nonlinearity, and transition ensues. Vortical structures fill the entire domain, and the nonlinear statistical steady states obtained resemble that of the turbulent nonrotating channel flow. This behavior os observed over a wide range of initial disturbance amplitudes, except at extremely low initial energy of the disturbance where the unstable mode emerges after the transients boost the disturbance energy, and the secondary flow grows exponentially until the nonlinear terms become important. Similar features in Görtler flow have also been observed where transition can be aided by the boosting of the unstable mode energy by algebraic mechanisms [54,55]. This suggests that algebraic nonmodal mechanisms are likely to be relevant even when exponential instabilities exist in certain parameter regimes due to the inherent nonnormal nature of the dynamics in shear flows.

On increasing the rotation rate to moderate levels $(R o \sim 0.2)$, the Coriolis force expectedly manifests itself in a more pronounced manner. Initial disturbances rapidly evolve into the most unstable eigenmode, and the resulting transitioned flow is strongly vortical with no characteristic structure or organization. This is in sharp contrast to the elongated structures seen at both lower and higher rotation rates. With further increase in rotation rate, the secondary (chaotic) flow is increasingly localized toward one wall, becoming smaller until it finally disappears. At high rotation rates, the base flow is extremely stable to nonmodal disturbances, as expected. At such values of $R o$, the effect of algebraic processes is negligible.

In summary, for the rotating channel flow, we have shown distinct behavior patterns at low, intermediate, and higher rotation rates, and the switchover between these is gradual with change in rotation rate. It is clear that algebraic growth can contribute decisively toward the transition in the rotating channel flow in a range of rotation rates.

\section{ACKNOWLEDGMENTS}

The Indo-French Centre for the Promotion of Advanced Research (IFCPAR)/Centre FrancoIndien pour la Promotion de la Recherche Avancée (CEFIPRA) is gratefully acknowledged for its financial support. S.J. would like to thank Prof. Luca Brandt at KTH Mechanics, Stockholm, for his help with understanding the SIMSON code. We are also grateful to the anonymous referees for their suggestions.

[1] A. E. Gill, Atmosphere-Ocean Dynamics, International Geophysics Series Vol. 30 (Academic Press, San Diego, USA, 1982).

[2] G. K. Vallis, Atmospheric and Oceanic Fluid Dynamics: Fundamentals and Large-Scale Circulation (Cambridge University Press, Cambridge, UK, 2006). 
[3] P. R. N. Childs, Rotating Flow (Elsevier, Oxford, UK, 2011).

[4] G. I. Taylor, Motion of solids in fluids when the flow is not irrotational, Proc. R. Soc. London, Ser. A 93, 99 (1917).

[5] J. Proudman, On the motion of solids in a liquid possessing vorticity, Proc. R. Soc. London, Ser. A 92, 408 (1916).

[6] P. Bradshaw, The analogy between streamline curvature and buoyancy in turbulent shear flow, J. Fluid Mech. 36, 177 (1969).

[7] T. J. Pedley, On the instability of viscous flow in a rapidly rotating pipe, J. Fluid Mech. 35, 97 (1969).

[8] J. E. Hart, Instability and secondary motion in a rotating channel flow, J. Fluid Mech. 45, 341 (1971).

[9] J. P. Johnston, R. M. Halleen, and D. K. Lezius, Effects of spanwise rotation on the structure of twodimensional fully developed turbulent channel flow, J. Fluid Mech. 56, 533 (1972).

[10] D. K. Lezius and J. P. Johnston, Roll-cell instabilities in rotating laminar and turbulent channel flows, J. Fluid Mech. 77, 153 (1976).

[11] P. H. Alfredsson and H. Persson, Instabilities in channel flow with system rotation, J. Fluid Mech. 202, 543 (1989).

[12] S. Yanase, C. Flores, O. Métais, and J. J. Riley, Rotating free-shear flows, I: Linear stability analysis, Phys. Fluids A 5, 2725 (1993).

[13] D. P. Wall and M. Nagata, Nonlinear secondary flow through a rotating channel, J. Fluid Mech. 564, 25 (2006).

[14] P. G. Drazin and W. H. Reid, Hydrodynamic Stability (Cambridge University Press, Cambridge, UK, 2004).

[15] S. Wallin, O. Grundestam, and A. V. Johansson, Laminarization mechanisms and extreme-amplitude states in rapidly rotating plane channel flow, J. Fluid Mech. 730, 193 (2013).

[16] W. H. Finlay, Transition to oscillatory motion in rotating channel flow, J. Fluid Mech. 215, 209 (1990).

[17] W. H. Finlay, Transition to turbulence in a rotating channel, J. Fluid Mech. 237, 73 (1992).

[18] K.-S. Yang and J. Kim, Numerical investigation of instability and transition in rotating plane Poiseuille flow, Phys. Fluids A 3, 633 (1991).

[19] M. Matsubara and P. H. Alfredsson, Secondary instability in rotating channel flow, J. Fluid Mech. 368, 27 (1998).

[20] D. P. Wall and M. Nagata, Three-dimensional exact coherent states in rotating channel flow, J. Fluid Mech. 727, 533 (2013).

[21] C. A. Daly, T. M. Schneider, P. Schlatter, and N. Peake, Secondary instability and tertiary states in rotating plane Couette flow, J. Fluid Mech. 761, 27 (2014).

[22] R. Kristoffersen and H. I. Andersson, Direct simulations of low-Reynolds-number turbulent flow in a rotating channel, J. Fluid Mech. 256, 163 (1993).

[23] E. Lamballais, O. Métais, and M. Lesieur, Spectral-dynamic model for large-eddy simulations of turbulent rotating channel flow, Theor. Comp. Fluid Dyn. 12, 149 (1998).

[24] O. Grundestam, S. Wallin, and A. V. Johansson, Direct numerical simulations of rotating turbulent channel flow, J. Fluid Mech. 598, 177 (2008).

[25] Z. Xia, Y. Shi, and S. Chen, Direct numerical simulation of turbulent channel flow with spanwise rotation, J. Fluid Mech. 788, 42 (2016).

[26] P. Yecko and M. Rossi, Transient growth and instability in rotating boundary layers, Phys. Fluids 16, 2322 (2004).

[27] P. A. Yecko, Accretion disk instability revisited-transient dynamics of rotating shear flow, Astron. Astrophys. 425, 385 (2004).

[28] G. D. Chagelishvili, J-P. Zahn, A. G. Tevzadze, and J. G. Lominadze, On hydrodynamic shear turbulence in Keplerian disks: Via transient growth to bypass transition, Astron. Astrophys. 402, 401 (2003).

[29] B. Mukhopadhyay, N. Afshordi, and R. Narayan, Growth of hydrodynamic perturbations in accretion disks: Possible route to non-magnetic turbulence, Adv. Space Res. 38, 2877 (2006).

[30] P. J. Schmid and D. S. Henningson, Stability and Transition in Shear Flows (Springer-Verlag, Berlin, 2001).

[31] P. J. Schmid, Nonmodal stability theory, Ann. Rev. Fluid Mech. 39, 129 (2007). 
[32] C. G. Speziale and S. Thangam, Numerical study of secondary flows and roll-cell instabilities in rotating channel flow, J. Fluid Mech. 130, 377 (1983).

[33] D. J. Tritton and P. A. Davies, Instabilities in geophysical fluid dynamics, in Hydrodynamic Instabilities and the Transition to Turbulence, edited by H. L. Swinney and J. P. Gollub (Springer, Berlin, 1985), pp. 229-270.

[34] S. Sipp, E. Lauga, and L. Jacquin, Vortices in rotating systems: Centrifugal, elliptic, and hyperbolic type instabilities, Phys. Fluids 11, 3716 (1999).

[35] R. C. Prima and G. J. Habetler, A completeness theorem for non-self-adjoint eigenvalue problems in hydrodynamic stability, Arch. Rat. Mech. Anal. 34, 218 (1969).

[36] J. A. Weideman and S. C. Reddy, A MATLAB differentiation matrix suite, ACM Trans. Math. Software 26, 465 (2000).

[37] D. D. Joseph, Stability of Fluid Motions I (Springer-Verlag, Berlin, 1976).

[38] D. D. Joseph and S. Carmi, Stability of Poiseuille flow in pipes, annuli, and channels, Q. Appl. Math. 26, 575 (1969).

[39] S. C. Reddy and D. S. Henningson, Energy growth in viscous channel flows, J. Fluid Mech. 252, 209 (1993).

[40] M. T. Landahl, A note on an algebraic instability of inviscid parallel shear flows, J. Fluid Mech. 98, 243 (1980).

[41] W. M`F. Orr, The stability or instability of the steady motions of a perfect liquid and of a viscous liquid. Part I: A perfect liquid, Proc. R. Irish Acad. A 27, 9 (1907).

[42] L. H. Gustavsson, Energy growth of three-dimensional disturbances in plane Poiseuille flow, J. Fluid Mech. 224, 241 (1991).

[43] C. A. Daly, Nonlinear and non-modal stability of structures evolving in shear flows, Ph.D. thesis, University of Cambridge, Cambridge, UK, 2014.

[44] S. Yanase and Y. Kaga, Zero-mean-absolute-vorticity state and vortical structures in rotating channel flow, J. Phys. Soc. Jpn. 73, 1419 (2004).

[45] M. Chevalier, P. Schlatter, A. Lundbladh, and D. S. Henningson, SIMSON: A pseudo-spectral solver for incompressible boundary layer flows, Tech. Rep. (KTH Mechanics, Stockholm, Sweden, 2007).

[46] S. C. Reddy, P. J. Schmid, J. S. Baggett, and D. S. Henningson, On stability of streamwise streaks and transition thresholds in plane channel flows, J. Fluid Mech. 365, 269 (1998).

[47] P. A. Elofsson, M. Kawakami, and P. H. Alfredsson, Experiments on the stability of streamwise streaks in plane Poiseuille flow, Phys. Fluids 11, 915 (1999).

[48] B. G. B. Klingmann, On transition due to three-dimensional disturbances in plane Poiseuille flow, J. Fluid Mech. 240, 167 (1992).

[49] T. Srikanth, H. N. Dixit, R. Tatavarti, and R. Govindarajan, Vortex shedding patterns, their competition, and chaos in flow past inline oscillating rectangular cylinders, Phys. Fluids 23, 073603 (2011).

[50] J. Jeong and F. Hussain, On the identification of a vortex, J. Fluid Mech. 285, 69 (1995).

[51] K. H. Bech and H. I. Andersson, Turbulent plane Couette flow subject to strong system rotation, J. Fluid Mech. 347, 289 (1997).

[52] E. Lamballais, M. Lesieur, and O. Métais, Effects of spanwise rotation on the vorticity stretching in transitional and turbulent channel flow, Int. J. Heat Fluid Flow 17, 324 (1996).

[53] M. Tanaka, S. Kida, S. Yanase, and G. Kawahara, Zero-absolute-vorticity state in a rotating turbulent shear flow, Phys. Fluids 12, 1979 (2000).

[54] L.-U. Schrader, L. Brandt, and T. A. Zaki, Receptivity, instability, and breakdown of Görtler flow, J. Fluid Mech. 682, 362 (2011).

[55] J.-M. Lucas, O. Vermeersch, and D. Arnal, Transient growth of Görtler vortices in two-dimensional compressible boundary layers: Application to surface waviness, Eur. J. Mech. B 50, 132 (2015). 